\title{
CUTOFF ON HYPERBOLIC SURFACES
}

\author{
KONSTANTIN GOLUBEV AND AMITAY KAMBER
}

\begin{abstract}
In this paper we study the common distance between points and the behavior of a constant length step discrete random walk on finite area hyperbolic surfaces. We show that if the second smallest eigenvalue of the Laplacian is at least $1 / 4$, then the distances on the surface are highly concentrated around the minimal possible value, and that the discrete random walk exhibits cutoff. This extends the results of Lubetzky and Peres ([20]) from the setting of Ramanujan graphs to the setting of hyperbolic surfaces. By utilizing density theorems of exceptional eigenvalues from [27], we are able to show that the results apply to congruence subgroups of $S L_{2}(\mathbb{Z})$ and other arithmetic lattices, without relying on the well known conjecture of Selberg ([28]).

Conceptually, we show the close relation between the cutoff phenomenon and temperedness of representations of algebraic groups over local fields, partly answering a question of Diaconis ([7]), who asked under what general phenomena cutoff exists.
\end{abstract}

\section{INTRODUCTION}

Let $\mathbb{H}$ be the hyperbolic plane equipped with the standard metric $d$ and the standard measure $\mu$. Let $\Gamma \subset P S L_{2}(\mathbb{R})$ be a lattice and let $X=\Gamma \backslash \mathbb{H}$ the the quotient space, which is a hyperbolic surface if $\Gamma$ is torsionfree, and an orbifold in general. The measure $\mu$ descends to a finite measure on $X$, and let $d_{X}: X \times X \rightarrow \mathbb{R}_{\geq 0}$ be the induced distance on $X$. The injectivity radius of a point $x_{0} \in X$ is $\frac{1}{2} \inf _{1 \neq \gamma \in \Gamma} d\left(\tilde{x}_{0}, \gamma \tilde{x}_{0}\right)$, where $\tilde{x}_{0} \in \mathbb{H}$ is a lift of $x_{0}$ to $\mathbb{H}$. Denote $R_{X}=\operatorname{acosh}(\mu(X) / 2 \pi+1)$. This is the radius of the hyperbolic ball whose volume equals the volume $\mu(X)$ of $X$.

Definition. We say that $X=\Gamma \backslash \mathbb{H}$ is Ramanujan ${ }^{1}$ if the non-trivial spectrum of the Laplacian on $L^{2}(X)$ is bounded from below by $1 / 4$. Equivalently, every non-trivial subrepresentation of $G$ on $L^{2}(\Gamma \backslash G)$ with $K=P S O_{2}(\mathbb{R})$-fixed vectors is tempered.

We write $C=C(t)$ if $C$ is a constant depending only on $t$. We write $a \ll_{t} b$ if there is $C=C(t)$ such that $a \leq C \cdot b$ holds, and $a \asymp_{t} b$ if both $a \ll_{t} b$ and $b \ll_{t} a$ take place.

\section{Common Distance.}

Theorem 1.1. Let $\Gamma \subset P S L_{2}(\mathbb{R})$ be a lattice, $X=\Gamma \backslash \mathbb{H}$, and assume $R_{X}=\operatorname{acosh}(\mu(X) / 2 \pi+1) \geq 1$. Then for a point $x_{0} \in X$ and for all $\gamma>0$, the following inequality holds

$$
\mu\left(x \in X: d_{X}\left(x_{0}, x\right) \leq R_{X}-\gamma \ln \left(R_{X}\right)\right) / \mu(X) \ll R_{X}^{-\gamma} .
$$

Konstantin Golubev, Bar-Ilan University and Weizmann Institute of Science, k.golubev@gmail.com Amitay Kamber, Einstein Institute of Mathematics, The Hebrew University of Jerusalem, amitay.kamber@mail.huji.ac.il.

${ }^{1}$ It seems that the notion of a Ramanujan surface (or more generally, a Ramanuajan manifold or a Ramanujan orbifold) does not appear in literature, but is natural given the standard notions of a Ramanujan graph ([21]) and a Ramanujan complex ([22]). 
If $X$ is Ramanujan and $x_{0} \in X$ has injectivity radius at least $r_{0}$, then for all $\gamma>0$, the following inequality holds

$$
\mu\left(x \in X: d_{X}\left(x_{0}, x\right) \geq R_{X}+\gamma \ln \left(R_{X}\right)\right) / \mu(X) \ll_{r_{0}}\left(1+\gamma^{2}\right) R_{X}^{2-\gamma} .
$$

In other words, for a point $x_{0}$ on a Ramanujan surface $X$, the distance from it to almost every other point is approximately $R_{X}$, with the window of size $(2+\epsilon) \ln \left(R_{X}\right)$. We emphasize that the constants in the theorem do not depend on the surface, and hence the result is interesting for a sequence of Ramanujan quotients with volume increasing to infinity, which is not known to exist. However, the well known conjecture of Selberg asserts that the quotients defined by the congruence subgroups of $S L_{2}(\mathbb{Z})$ form a sequence of such quotients (see $[28,26]$ and also Theorem 1.3 below). Alternatively, one may conjecture that as in case of graphs, a "random" surface is almost Ramanujan with a proper choice of the random model (see Conjecture 1.5 below).

Cutoff of Random Walks. In the second result, we consider the speed of convergence in the $L^{1}$-norm of two different random walks on $X$. The first one is the hyperbolic Brownian motion on $X$, which we consider as an operator $B_{t}: C(X) \rightarrow C(X)$ for $t \in \mathbb{R}_{\geq 0}$, where $C(X)$ is the space of continuous functions on $X$. The second one is the discrete time random walk with step of a fixed length, i.e., at each step the walker rotates at a uniformly chosen angle and makes a step of some fixed length $r_{1}>0$. The corresponding operator $A_{r_{1}}: C(X) \rightarrow C(X)$ is the distance $r_{1}$ averaging operator. By duality, we consider both random walks as acting on measures on $X$.

Specifically, for a point $x_{0} \in X$ consider the continuous time random walk $B_{t} \delta_{x_{0}}$, and the discrete time random walk $A_{r_{1}}^{k} \delta_{x_{0}}$, both considered as measures on $X$. One can show that the measures defined by the two random walks, for $t \gg 0$ or $k \gg 0$, are defined by some $L^{1}$-functions, which converge in the $L^{1}$-norm to the constant function $\pi$ on $X$ normalized as $\pi(x)=\mu(X)^{-1}$ for all $x \in X$. The following theorem gives an exact estimate on the rate of convergence for points with injectivity radius bounded away from 0 .

Theorem 1.2. Fix $0<r_{0}, 0<r_{1}$, assume that $X=\Gamma \backslash \mathbb{H}$ is Ramanujan, and let $x_{0} \in X$ be a point with injectivity radius at least $r_{0}$.

(1) There exist constants $c=c\left(r_{1}\right)>0$, and $C=C\left(r_{0}, r_{1}\right)$, such that

(a) If $k$ satisfies $k \alpha r_{1}<R_{X}-\lambda \sqrt{R_{X}}$ then $\left\|A_{r_{1}}^{k} \delta_{x_{0}}-\pi\right\|_{1}>2-C e^{-c \lambda^{2}}$;

(b) If $k$ satisfies $k \alpha r_{1}>R_{X}+\lambda \sqrt{R_{X}}$ then $\left\|A_{r}^{k} \delta_{x_{0}}-\pi\right\|_{1}<C e^{-c \lambda^{2}}$;

for every $\lambda>0$, assuming $R_{X} \gg_{r_{0}, r_{1}, \lambda} 1$, and where $\alpha=\frac{1}{\pi r_{1}} \int_{0}^{\pi} \ln \left(e^{r_{1}} \cos ^{2} \theta+e^{-r_{1}} \sin ^{2} \theta\right) d \theta \in$ $(0,1)$.

(2) There exist constants $c>0, C=C\left(r_{0}\right)$ such that

(a) If $t$ satisfies $t<R_{X}-\lambda \sqrt{R_{X}}$ then $\left\|B_{t} \delta_{x_{0}}-\pi\right\|_{1}>2-C e^{-c \lambda^{2}}$;

(b) If $t$ satisfies $t>R_{X}+\lambda \sqrt{R_{X}}$ then $\left\|B_{t} \delta_{x_{0}}-\pi\right\|_{1}<C e^{-c \lambda^{2}}$;

for every $\lambda>0$, assuming $R_{X} \gg_{r_{0}, \lambda} 1$.

As in Theorem 1.1, the lower bounds $(1 a)$ and $(2 a)$ do not exploit the assumption that $X$ is Ramanujan nor the assumption that the injectivity radius of $x_{0}$ exceeds $r_{0}$.

The above behavior of the random walk is closely related to the cutoff phenomenon, which is defined in general as follows (see [7]). Let $\left(P_{n}(x, y), X_{n}\right)$ be a series of Markov random walks on a probability space $X_{n}$, and let $P_{n}^{t}(x, y)$ the $t$-step random walk on $X_{n}$. Let $f(n), g(n)$ be functions such that $f(n)$ tends to 
infinity and $g(n)=o(f(n))$ as $n \rightarrow \infty$. We say that the series $\left(P_{n}(x, y), X_{n}\right)$ exhibits a cutoff at time $f(n)$ with window of size $g(n)$, if for every $1>\epsilon>0$, the time $t_{n}=\inf \left\{t \mid \sup _{x_{0}}\left\|P_{n}^{t}\left(x_{0}, \cdot\right)-\pi_{n}\right\|_{1}<\epsilon\right\}$ satisfies $t_{n}=f(n)+O_{\epsilon}(g(n))$. Determining whether a series of random walks exhibit a cutoff is a fundamental problem (see [7]). Theorem 1.2 says that if a sequence of surfaces $X_{n}$ have injectivity radius at least $r_{0}$ at every point of every surface then the random walks on them exhibit a cutoff (and moreover the mixing time from each point is the same and can be estimated explicitly).

Arithmetic Subgroups. As said, Selberg's conjecture implies that the quotients $X$ of $\mathbb{H}$ by congruence subgroups of $S L_{2}(\mathbb{Z})$ satisfy the results of Theorem 1.1 and Theorem 1.2. Using the corrent knowledge, we can give slightly weaker statements (at least for Theorem 1.1), which capture the essence of the result.

Theorem 1.3. Let $\Gamma=S L_{2}(\mathbb{Z})$ or any cocompact arithmetic lattice in $S L_{2}(\mathbb{R}), X_{0}=\Gamma \backslash \mathbb{H}$ the corresponding quotient, $q \in \mathbb{N}, \Gamma(q)$ the principal congruence subgroup of $\Gamma, X_{q}=\Gamma(q) \backslash \mathbb{H}$ the corresponding quotient, and $\rho_{q}: X_{q} \rightarrow X_{0}$ be the cover map.

Let $x_{0}^{(q)} \in X_{q}$ be a point such that its projection $\rho_{q}\left(x_{0}^{(q)}\right)$ to $X_{0}$ has injectivity radius at least a constant $r_{0}$. Then for every $\epsilon_{0}>0$

$$
\mu\left(x \in X_{q}: d_{X_{q}}\left(x, x_{0}^{(q)}\right) \geq R_{X_{q}}\left(1+\epsilon_{0}\right)\right) / \mu\left(X_{q}\right) \rightarrow_{q \rightarrow \infty} 0 .
$$

Methods of Proof. The proofs of the three Theorems exploit the following proposition:

Proposition 1.4. The surface $X$ is Ramanujan if and only if for every $r \geq 0$ the non-trivial spectrum of $A_{r}$ on $L_{0}^{2}(X)=\left\{f \in L^{2}(X): \int f(x) d x=0\right\}$ is bounded by $(r+1) e^{-r / 2}$.

A similar (generalized) proposition plays a crucial role in the work of Harish-Chandra (see [10, Theorem 3]). Theorem 1.1 is actually a direct application of Proposition 1.4.

The proof of Theorem 1.2 combines Proposition 1.4 with two other results. The first one, Proposition 5.4, says that after 3 steps the random walk measure $A_{r_{1}}^{3} \delta_{x_{0}}$ (respectively, the Brownian motion measure $B_{t_{0}} \delta_{x_{0}}$ at a fixed time $t_{0}>0$ ) is an $L^{2}$ function on $X$, with a bounded $L^{2}$ norm depending only on the injectivity radius $r_{0}$. The second result, Proposition 4.7 and Proposition 4.8, which is well known for the Brownian motion, may be described as a concentration of measure theorem for the rate of escape of the random walk $A_{r_{1}}^{k}$ (respectively $\left.B_{t}\right)$ on $\mathbb{H}$. Namely, we may write $A_{r_{1}}^{k} \cong \int_{r} f_{k}(r) A_{r} d r$ (respectively $\left.B_{t} \cong \int_{r} g_{t}(r) A_{r} d r\right)$, where most of the measure $f_{k}(r) d r$ is concentrated at $\sim \alpha k r_{1}$ (respectively, $g_{t}(r) d r$ is concentrated at $\sim t$ ).

The proof of Theorem 1.3 depends on the following facts: $\Gamma(q)$ is normal in $\Gamma$, there exists an absolute lower bound on the smallest eigenvalue of $X_{q}$, on a careful general analysis of the required bounds on the number of exeptional eigenvalues of $X_{q}$, and on an $L_{p}$ generalization of Proposition 1.4. It is a beautiful result that the bound of the number of exceptional eigenvalues that is required is exacly the "elementary" density bound discussed in [27]. The bound states the number of eigenvalues of $X_{q}$ with corresponding matrix coefficients not in $L^{p}$ for $p>2$ is $\ll_{\epsilon}[\Gamma: \Gamma(q)]^{2 / p+\epsilon}$ (see also [25]). Note that the article [27] assumes cocompactness, which was removed in [13] (stronger results for $S L_{2}(\mathbb{Z})$ were also proven earlier by different methods in $[15,14])$. Theorem 1.3 also holds for $S L_{2}(\mathbb{Z})$ for non-prime $q$, as a non-elementary bound on the smallest eigenvalue was proven already by Selberg in [28]. See the discussion in Section 8 for full details.

This work is similar in spirit to the results of [20], and shows the general connection between the common distance and cutoff phenomena in quotients of symmetric spaces (infinite regular trees and the hyperbolic plane in these cases) and temperedness of representations (or the Ramanujan conjecture). 
Open questions. We expect that the results of this article can be extended to quotients of higher dimensions, and also to other contexts (e.g. the action of Hecke operators on $S L_{2}(Z) \backslash S L_{2}(\mathbb{R})$ and its covers). Theorems analogous to Theorem 1.1 for quotients of $p$-adic Lie groups (i.e. Ramanujan complexes) are proven in [16, Theorem 1.9], and [19, Theorem 1.ii]. Theorem 1.1 is also closely related to the optimal covering properties of the Golden-Gates of [24].

While we were unable to show it, we believe that it is possible to prove in the notations of Theorem 1.3 that (at least for $S L_{2}(\mathbb{Z})$ ) there exists a constant $C>0$ such that

$$
\mu\left(x \in X_{q}: d_{X_{q}}\left(x, x_{0}^{(q)}\right) \geq R_{X_{q}}+C \ln \left(R_{X_{q}}\right)\right) / \mu\left(X_{q}\right) \rightarrow_{q \rightarrow \infty} 0 .
$$

Selberg's conjecture would give $C=2+\epsilon, \epsilon>0$ by Theorem 1.1. The stronger density theorems of [15] fall just a bit short of proving it. See Remark 8.4.

The following conjectures are natural continuous analogs of well known combinatorial results, in the spirit of this article. Assume that the lattice $\Gamma$ is a free group (for example, the principal congruence subgroup $\Gamma=$ $\Gamma(2)=\operatorname{ker}\left\{P S L_{2}(\mathbb{Z}) \stackrel{\bmod }{\rightarrow} P S L_{2}(\mathbb{Z} / 2 \mathbb{Z})\right\}$, which is freely generated by $\left(\begin{array}{ll}1 & 2 \\ 0 & 1\end{array}\right)$ and $\left.\left(\begin{array}{ll}1 & 0 \\ 2 & 1\end{array}\right)\right)$. Then every onto homomorphism $\phi: \Gamma \rightarrow S_{n}$ defines an index $n$ subgroup $\Gamma^{\prime} \subset \Gamma$, by $\Gamma^{\prime}=\{\gamma \in \Gamma: \phi(\gamma)(1)=1\}$, and every index $n$ subgroup of $\Gamma$ can be defined this way. Since each homomorphism is defined using the generators, there is a finite number of such homomorphism, and it defines a probability measures on the index $n$ subgroups of $\Gamma$, or equivalently, the $n$-covers of $X$.

Conjecture 1.5. Assume that $\Gamma$ is a free group.

(1) For every $\epsilon>0$, the probability that every new eigenvalue $\lambda$ of an $n$-cover $X^{\prime}$ of $X$ satisfies $\lambda \geq 1 / 4+\epsilon$ is $1-o(1)$.

An analogous statement for graphs is called Alon's conjecture, and was proved in [8].

(2) There exists a 2-cover $X^{\prime}$ of $X$, such that every new eigenvalue $\lambda$ of $X^{\prime}$ satisfies $\lambda \geq 1 / 4$.

In the graph setting this statement is called Bilu-Linial's conjecture, and was solved for the bipartite case in [23].

See also [1], where (in a slightly different random model) weaker versions of (1) are proved.

Outline of the Article. In Section 2 we set notations and discuss the harmonic analysis on $\mathbb{H}$, and its relation to the operator $A_{r}$ and the Laplacian. We also prove a bound on the $L^{2}$-spectrum of $A_{r}$ on $\mathbb{H}$. In Section 3 we prove Theorem 1.1. In Section 4 we prove some versions of the central limit theorem for the random walks. For the discrete random walk we reduce the problem to the standard central limit theorem. For the Brownian motion this result is well known. In Section 5 we prove that after a short time the random walks sends the delta measure on a point to a bounded $L^{2}$-function. In Section 6 we prove Theorem 1.2.

In the rest of the article we prove a generalized version of Theorem 1.3. In Section 7 we generalize the bounds for spectra and Proposition 1.4 to the non-Ramanujan case. We also give a weak version of Theorem 1.1, which depends on the smallest non-trivial eigenvalue of the Laplacian. In Section 8 we discuss covers of a fixed quotient $X_{0}$, and in particular normal covers. The requirement on the spectrum of normal covers is stated somewhat abstractly in Theorem 8.1. We then discuss density theorems and known results about them, and show that the density theorems satisfy the requirements of Theorem 8.1, thus proving Corollary 8.3, which implies Theorem 1.3. 
We also have two appendices. In the first appendix, Section 9, we prove that for any fixed $x_{0} \in X$ there exists a distance $R_{x_{0}, X}$ such that the distances from $x_{0}$ to other vertices is concentrated around $R_{x_{0}, X}$ in a window of a constant size, where the constant depends on the smallest non-trivial eigenvalue of the Laplacian. Theorem 1.1 implies that if $X$ is Ramanujan and $x_{0}$ has a lower bound on its injectivity radius, then $R_{X} \leq R_{X, x_{0}} \leq R_{X}+(2+\epsilon) \ln R_{X}$. The proof involves some interesting isoperimetric inequalities.

In the second appendix, Section 10, we show that the Gaussian random walks on the flat surfaces $\left(a \mathbb{Z}^{2}\right) \backslash \mathbb{R}^{2}$, $a \rightarrow \infty$ do not exhibit a cutoff.

Acknowledgments. We are grateful to Elon Lindenstrauss, Alex Lubotzky, Shachar Mozes and Józef Dodziuk for fruitful discussions. The first author is supported by the ERC grant 336283. This work is part of the Ph.D. thesis of the second author at the Hebrew University of Jerusalem. A large part of this work was carried out in the café Bread\&Co in Tel-Aviv, to which we are thankful for its coffee and hospitality.

\section{Preliminaries}

The hyperbolic plane. There are several models for the hyperbolic plane $\mathbb{H}$ of constant curvature -1 , and we stick to the upper half-plane model. That is the complex half-plane $\{z \in \mathbb{C} \mid \operatorname{Im}(z)>0\}$ endowed with the metric $d s^{2}=d z^{2} /(\operatorname{Im}(z))^{2}$. For $z=x+i y, z^{\prime}=x^{\prime}+i y^{\prime} \in \mathbb{H}$ the distance $d\left(z, z^{\prime}\right)$ between them is

$$
d\left((x, y),\left(x^{\prime}, y^{\prime}\right)\right)=\operatorname{acosh}\left(1+\frac{\left(x^{\prime}-x\right)^{2}+\left(y^{\prime}-y\right)^{2}}{2 y y^{\prime}}\right) .
$$

The group $G=P S L_{2}(\mathbb{R})$ acts on $\mathbb{H}$ by Mobius transformations, i.e.,

$$
\left(\begin{array}{ll}
a & b \\
c & d
\end{array}\right) \cdot z=\frac{a z+b}{c z+d}
$$

and constitutes the group of orientation preserving isometries of $\mathbb{H}$. It also acts transitively on the points of $\mathbb{H}$, with the subgroup $K=P S O_{2}(\mathbb{R}) \subset G$ being the stabilizer of the point $i$, to which we refer as the origin of $\mathbb{H}$. The subgroup $K$ acts on $\mathbb{H}$ by rotations around $i$. The plane $\mathbb{H}$ can be identified with the quotient $G / K$, and in particular, the circle of radius $r$ around $i$ identifies with the double coset $K\left(\begin{array}{cc}e^{r / 2} & 0 \\ 0 & e^{-r / 2}\end{array}\right) K$. The Haar measure on $G$ which is normalized so that the measure of $K$ is equal to 1 agrees with the standard measure $\mu$ on $\mathbb{H}$.

Harmonic analysis on $\mathbb{H}$. For $f \in L^{1}(\mathbb{H})$, its Helgason-Fourier transform $\widehat{f}(s, k) \in C(\mathbb{C} \times K)$, is defined for $s \in \mathbb{C}$ and $k \in K=\mathrm{PSO}_{2}(\mathbb{R})$ as

$$
\widehat{f}(s, k)=\int_{\mathbb{H}} f(z) \overline{(\operatorname{Im}(k z))^{\frac{1}{2}+i s}} d z .
$$

In the case when $f$ is $K$-invariant, i.e., $f(k z)=f(z)$ for all $z \in \mathbb{H}$ and $k \in K$, its transform is independent of $k$ and can be written with the help of the spherical functions. For every $s \in \mathbb{C}$, the corresponding spherical function is a $K$-invariant function defined as

$$
\varphi_{\frac{1}{2}+i s}(z)=\int_{K} \overline{\left(\operatorname{Im}(k z)^{\frac{1}{2}+i s-2}\right.} d k .
$$


Since $\varphi_{\frac{1}{2}+i s}$ is $K$-invariant, it depends solely on the distance from a point to the origin $i$, and can be written as

$$
\varphi_{\frac{1}{2}+i s}(z)=\varphi_{\frac{1}{2}+i s}\left(k e^{-r} i\right)=P_{-\frac{1}{2}+i s}(\cosh r),
$$

where $k \in K, r \in \mathbb{R}_{\geq 0}$ is the distance from $z$ to $i$, and $P_{s}(r)$ is the Legendre function of the first kind. Then the Helgason-Fourier transform of a $K$-invariant function $f$ reads as

$$
\widehat{f}(s)=\int_{\mathbb{H}} f(z) \varphi_{\frac{1}{2}+i s}(z) d z=\int_{0}^{\infty} f\left(e^{-r} i\right) P_{-\frac{1}{2}+i s}(\cosh r) \sinh r d r .
$$

For two functions $f_{1}, f_{2} \in L^{1}(\mathbb{H})$, their convolution is defined as

$$
f_{1} * f_{2}(z)=\int_{G} f_{1}(g i) f_{2}\left(g^{-1} z\right) d g .
$$

We exploit of the following properties of the Helgason-Fourier transform on $\mathbb{H}$. For an extensive presentation of the theory, see [11, 29].

Proposition 2.1. ([29, Theorem 3.2.3])

(1) (Plancherel Formula) The map $f \rightarrow \widehat{f}$ extends to an isometry of $L^{2}(\mathbb{H}, d \mu)$ with $L^{2}\left(\mathbb{R} \times K, \frac{1}{4 \pi} s \tanh \pi s d s d k\right)$, where the $K$ is identified with the interval $[0,1)$.

(2) (Convolution property) For $f, g \in L^{1}(\mathbb{H})$, where $g$ is $K$-invariant,

$$
\widehat{f * g}=\widehat{f} \cdot \widehat{g},
$$

where $*$ stands for convolution, and $\cdot$ for pointwise multiplication.

The Helgason-Fourier transform can be extended to compactly supported measures on $\mathbb{H}$. Namely, for such a measure $\nu$, its transform $\widehat{f}(s, k) \in C(\mathbb{C} \times K)$, is defined for $s \in \mathbb{C}$ and $k \in K=P_{S O_{2}}(\mathbb{R})$ as

$$
\widehat{\nu}(s, k)=\int_{\mathbb{H}} \overline{(\operatorname{Im}(k(z)))^{\frac{1}{2}+i s}} d \nu,
$$

and, if the measure is $K$-invariant, its transform is independent of $k$, and can be written as

$$
\widehat{\rho}(s)=\int_{\mathbb{H}} \varphi_{\frac{1}{2}+i s}(z) d \rho .
$$

We will need the following claim, which follows from Theorem 2.1.

Corollary 2.2. Let $\nu$ be a compactly supported distribution on $\mathbb{H}$, and assume that $\widehat{\nu} \in L^{2}\left(\mathbb{R} \times K, \frac{1}{4 \pi} s \tanh \pi s d t d k\right)$. Then $\nu$ can be represented as an $L^{2}$ function on $\mathbb{H}$, i.e. there exists $f_{\nu} \in L^{2}(\mathbb{H})$ such that for every $f \in C_{c}(\mathbb{H})$, $\nu(f)=\int f_{\nu}(x) f(x) d x$.

The averaging operator $A_{r}$. For $r>0$, let $A_{r}$ denote the operator on $C(\mathbb{H})$ which averages a function over a circle of radius $r$, i.e., for a function $f \in C(\mathbb{H})$ and $z \in \mathbb{H}$

$$
\left(A_{r} f\right)(z)=\int_{K} f\left(k\left(\begin{array}{cc}
e^{r / 2} & 0 \\
0 & e^{-r / 2}
\end{array}\right) z\right) d k .
$$

The operator $A_{r}$ is bounded and self adjoint with respect to the $L^{2}$-norm on $L^{2}(\mathbb{H}) \cap C(\mathbb{H})$, so it extends to an operator $A_{r}: L^{2}(\mathbb{H}) \rightarrow L^{2}(\mathbb{H})$, which is also self adjoint. By duality, we may also extend $A_{r}$ to an 
operator on compactly supported measures on $\mathbb{H}$. Note that the operator $A_{r}$ can written as a convolution with a uniform $K$-invariant probability measure $\delta_{S_{r}}$ supported on the double coset $K\left(\begin{array}{rr}e^{r / 2} & 0 \\ 0 & e^{-r / 2}\end{array}\right) K$.

Note that the Laplace-Beltrami operator $\Delta=-y^{2}\left(\frac{\partial^{2}}{\partial x^{2}}+\frac{\partial^{2}}{\partial y^{2}}\right)$ can be written on $C^{\infty}(\mathbb{H})$ as the limit

$$
\Delta=(-2) \lim _{r \rightarrow 0} \frac{1}{r^{2}}\left(I-A_{r}\right)
$$

where $I$ stands for the identity operator. However, we are mainly concerned with the behavior of $A_{r}$ when $r$ is either fixed or approaches infinity.

The spherical functions $\varphi_{\frac{1}{2}+i s}$ on $\mathbb{H}$ are eigenfunctions of $\Delta$ and of $A_{r}$ for every $r>0$, namely,

$$
\begin{aligned}
\Delta \varphi_{\frac{1}{2}+i s} & =\left(\frac{1}{4}+s^{2}\right) \varphi_{\frac{1}{2}+i s} \\
A_{r} \varphi_{\frac{1}{2}+i s} & =\varphi_{\frac{1}{2}+i s}\left(e^{-r} i\right) \cdot \varphi_{\frac{1}{2}+i s} .
\end{aligned}
$$

In particular, it follows from Proposition 2.1 that the $L^{2}$-spectrum of $\Delta$ on $\mathbb{H}$ is $\left[\frac{1}{4}, \infty\right)$ and the $L^{2}$-spectrum of $A_{r}$ on $\mathbb{H}$ is the set $\left\{\varphi_{\frac{1}{2}+i s}\left(e^{-r} i\right) \mid s \in \mathbb{R}\right\}=\left\{P_{-\frac{1}{2}+i s}(\cosh r) \mid s \in \mathbb{R}\right\}$.

Spectrum on the Quotients and the Ramanujan condition. Consider the actions of $A_{r}$ and of $\Delta$ on a dense subspace of $L_{0}^{2}(\Gamma \backslash \mathbb{H})=\left\{f \in L^{2}(\Gamma \backslash \mathbb{H}): \int f=0\right\}$, where $\Gamma \subseteq P S L_{2}(\mathbb{R})$ is a lattice. In both cases the spectrum is not necessarily discrete, but is parameterized by the unitary dual parameter $\frac{1}{2}+i s \in \mathbb{C}$. Namely, if $\frac{1}{2}+i s \in \mathbb{C}$ appears in the unitary dual of $X=\Gamma \backslash \mathbb{H}$, then $P_{-\frac{1}{2}+i s}(\cosh r)$ is in the spectrum of $A_{r}$ and $\frac{1}{4}+s^{2}$ is an eigenvalue of the Laplacian. It is well known that, in general, the unitary dual is contained in the set $\left\{\frac{1}{2}+i s \mid s \in \mathbb{R}\right\} \cup\left\{\frac{1}{2}+i s \mid i s \in\left(-\frac{1}{2}, \frac{1}{2}\right)\right\} \cup\{0,1\}$, where the first set is called the principal series, the second one is called the complementary series, and $\{0,1\}$ is called trivial. The trivial part corresponds to the constant function on $X$. A quotient $X=\Gamma \backslash \mathbb{H}$ is called Ramanujan if its non-trivial unitary dual is contained solely in $\left\{\frac{1}{2}+i s \mid s \in \mathbb{R}\right\}$. Equivalently, $X$ is Ramanujan iff all the non-trivial eigenvalues of the Laplacian are greater or equal to $\frac{1}{4}$.

Another equivalent condition of being a Ramanujan quotient is that the subrepresentation of $G$ on $L_{0}^{2}(\Gamma \backslash G)$ generated by its $K=P S O_{2}(\mathbb{R})$ fixed vectors, is tempered. This statement can also be stated as follows. Every function $f$ on $X$ can be lifted to a $\Gamma$-invariant function $\tilde{f}$ on $\mathbb{H}$. Then $X$ is Ramanujan iff for every $f, f^{\prime} \in L_{0}^{2}(X)$, and for every $\epsilon>0$,

$$
\int_{G}\left|\left\langle\tilde{f}, g \tilde{f}^{\prime}\right\rangle\right|^{2+\epsilon} d g<\infty
$$

\section{Harish-Chandra Bounds.}

Proposition 2.3. The spectrum of $A_{r}$ on $L^{2}(\mathbb{H})$ is bounded by $(r+1) e^{-r / 2}$.

Proof. The $L^{2}$-spectrum is composed of eigenvalues of $A_{r}$ on the principal series spherical functions, and hence is equal to $\left\{P_{-\frac{1}{2}+i s}(\cosh r)\right\}_{s \in \mathbb{R}}$. Alternatively, it can be written as the range of the function $\phi_{\frac{1}{2}+i s}(r)=$ $\frac{\sqrt{2}}{\pi} r \int_{0}^{1} \frac{\cos (s r x)}{\sqrt{\cosh r-\cosh r x}} d x$, for $s \in \mathbb{R}([5$, Lemma 7], or [29, Exercise 3.2.28], ). Since $\cosh r-\cosh (r x) \geq$ 
$(\cosh r-1)\left(1-x^{2}\right)$, for $0 \leq x \leq 1$, (which follows from the Taylor expansion of $\cosh$ ), the following inequalities hold

$$
\begin{aligned}
\left|\phi_{\frac{1}{2}+i s}(r)\right| & =\frac{\sqrt{2}}{\pi} r\left|\int_{0}^{1} \frac{\cos (s r x)}{\sqrt{\cosh r-\cosh r x}} d x\right| \leq \frac{\sqrt{2}}{\pi} r \frac{1}{\sqrt{\cosh r-1}} \int_{0}^{1} \frac{1}{\sqrt{1-x^{2}}} d x \\
& \leq \frac{\sqrt{2}}{\pi} r \frac{1}{\sqrt{\cosh r-1}} \int_{0}^{1} \frac{1}{\sqrt{1-x^{2}}} d x=\frac{1}{\sqrt{2}} r(\cosh r-1)^{-1 / 2}=\frac{r}{2}\left(\sinh \frac{r}{2}\right)^{-1} \leq(r+1) e^{-r / 2} .
\end{aligned}
$$

Corollary 2.4. If $X$ is Ramanujan then the norm of $A_{r}$ on $L_{0}^{2}(X)$ is bounded by $(r+1) e^{-r / 2}$.

The inverse direction can be proven in a similar way, by analyzing the complementary series. Let us present a more conceptual proof of it:

Proposition 2.5. If for every $r \geq 0$ the norm of $A_{r}$ on $L_{0}^{2}(X)$ is bounded by $(r+1) e^{-r / 2}$ then $X$ is Ramanujan.

Proof. Recall that the condition on the Laplacian is equivalent to the fact that the subrepresentation of $G$ on $L_{0}^{2}(\Gamma \backslash G)$ with $K=P S O_{2}(\mathbb{R})$ fixed vectors, is tempered. Consider the Cartan decomposition $G=$

$\cup_{r \geq 0} K\left(\begin{array}{cc}e^{r} & 0 \\ 0 & e^{-r}\end{array}\right) K$, which corresponds to the polar coordinates in $\mathbb{H}$. The metric on the group in this coordinates reads as $d g=\sinh r d k^{\prime} d k d r$. Let $f, f^{\prime} \in L_{0}^{2}(X)$ and let $\tilde{f}, \tilde{f}^{\prime} \in L_{0}^{2}(\Gamma \backslash G)$ be their lifts. Then

$$
\begin{aligned}
\int_{G}\left|\left\langle\tilde{f}, g \tilde{f}^{\prime}\right\rangle\right|^{2+\epsilon} d g & =\int_{r \geq 0} \sinh r\left|\left\langle\tilde{f},\left(\begin{array}{cc}
e^{r} & 0 \\
0 & e^{-r}
\end{array}\right) \tilde{f}^{\prime}\right\rangle\right|^{2+\epsilon} d r= \\
& =\int_{r \geq 0} \sinh r\left|\left\langle f, A_{r} f^{\prime}\right\rangle\right|^{2+\epsilon} d r
\end{aligned}
$$

Using the fact that for $r$ large $\sinh r \asymp e^{r}$, we see that the Ramanujan condition is equivalent to the condition:

- For every $f, f^{\prime} \in L_{0}^{2}(X)$ and for every $\epsilon>0, \int_{r \geq 0} e^{r}\left|\left\langle f, A_{r} f^{\prime}\right\rangle\right|^{2+\epsilon} d r<\infty$

If the inequality holds then $\left|\left\langle f, A_{r} f^{\prime}\right\rangle\right| \leq(r+1) e^{-r / 2}\left|\left\langle f, f^{\prime}\right\rangle\right|$ for every positive $r$, so

$$
\begin{aligned}
\int_{r \geq 0} e^{r}\left|\left\langle f, A_{r} f^{\prime}\right\rangle\right|^{2+\epsilon} d r & \leq \int_{r \geq 0} e^{r} e^{(-1-\epsilon / 2) r}(r+1)^{2+\epsilon}\left|\left\langle f, f^{\prime}\right\rangle\right|^{2+\epsilon} d r= \\
& =\left|\left\langle f, f^{\prime}\right\rangle\right|^{2+\epsilon} \int_{r \geq 0}(r+1)^{2+\epsilon} e^{-\epsilon r} d r<\infty,
\end{aligned}
$$

and the proposition follows.

\section{Proof of Theorem 1.1}

Proof. of Theorem 1.1. Let $r \leq R_{X}-\gamma \ln \left(R_{X}\right)$. The measure of $Y_{<}=\left\{x \in X: d\left(x, x_{0}\right)<r\right\}$ is at most the volume of the ball of radius $r$ in the hyperbolic plane, i.e.,

$$
\mu\left(Y_{<}\right) \leq \mu\left(B_{r}\right) \ll e^{r}=e^{R_{X}} e^{-\gamma \ln \left(R_{X}\right)} \ll \mu(X) R_{X}^{-\gamma},
$$

which implies the lower bound of the theorem (note that we assume that $\mu(X) \asymp e^{R_{X}}$ since $R_{X} \geq 1$ ). 
Now let $r^{\prime}=R_{X}+\gamma \ln \left(R_{X}\right)-r_{0}$, and $Y_{>}=\left\{x \in X: d_{X}\left(y, x_{0}\right)>r^{\prime}\right\}$. Let $b_{x_{0}, r_{0}}$ be the characteristic function of $B_{x_{0}}\left(r_{0}\right) \subset X$, normalized as follows:

$$
b_{x_{0}, r_{0}}(x)= \begin{cases}1 / \mu\left(B_{r_{0}}\right), & x \in B_{x_{0}}\left(r_{0}\right) \\ 0, & x \notin B_{x_{0}}\left(r_{0}\right) .\end{cases}
$$

It is well defined since $x_{0}$ has injectivity radius at least $r_{0}$. Then $Y>Z$ where $Z=\left\{x \in X: A_{r^{\prime}} b_{x_{0}, r_{0}}(x)=0\right\}$. Denote $\pi \in L^{2}(X)$ the constant function with $\pi(x)=1 / \mu(X)$ for every $x \in X$. For every point $x \in Z$, one has $\left|\left(A_{r^{\prime}} b_{x_{0}, r_{0}}-\pi\right)(x)\right|=\pi(x)=\frac{1}{\mu(X)}$, so $\mu(Z) \mu^{-2}(X) \leq\left\|A_{r^{\prime}} b_{x_{0}, r_{0}}-\pi\right\|_{2}^{2}$. Therefore $\mu\left(Y_{>}\right) \leq \mu(Z) \leq$ $\mu^{2}(X)\left\|A_{r_{n}^{\prime}} b_{x_{0}, r_{0}}-\pi\right\|_{2}^{2}$.

Since $b_{x_{0}, r_{0}}-\pi \perp \pi$ in the space $L^{2}(X)$, it holds that

$$
\left\|b_{x_{0}, r_{0}}-\pi\right\|_{2} \leq\left\|b_{x_{0}, r_{0}}\right\|_{2}=\mu\left(B_{r_{0}}\right)^{-1 / 2} \ll_{r_{0}} 1 .
$$

The bounds on the norm of $A_{r^{\prime}}$ of Proposition 1.4 imply the following inequality

$$
\begin{aligned}
\left\|A_{r^{\prime}} b_{x_{0}, r_{0}}-\pi\right\|_{2} & =\left\|A_{r^{\prime}}\left(b_{x_{0}, r_{0}}-\pi\right)\right\|_{2} \leq\left(r^{\prime}+1\right) e^{-r^{\prime} / 2}\left\|b_{x_{0}, r_{0}}-\pi\right\|_{2} \\
& \ll_{r_{0}}\left(\frac{R_{X}+\gamma \ln \left(R_{X}\right)-r_{0}+1}{R_{X}}\right) R_{X} e^{-\frac{1}{2} R_{X}-\frac{1}{2} \gamma \ln \left(R_{X}\right)+\frac{1}{2} r_{0}} \\
& \ll_{r_{0}}(1+\gamma) e^{-R_{X} / 2} R_{X}^{1-\gamma / 2} \ll(1+\gamma) \mu(X)^{-1 / 2} R_{X}^{1-\gamma / 2} .
\end{aligned}
$$

And he following inequality completes the proof

$$
\mu\left(Y_{>}\right) \leq \mu^{2}(X)\left\|A_{r^{\prime}} b_{x_{0}, r_{0}}-\pi\right\|_{2}^{2} \ll_{r_{0}} \mu(X)\left(1+\gamma^{2}\right) R_{X}^{2-\gamma} .
$$

\section{Deviations of the Random Walk}

Let $r_{1}>0$ be fixed. Consider the random walk on $\mathbb{H}$, emanating from $z_{0}=i$ and having $z_{k+1}$ equidistributed on the sphere of radius $r_{1}$ around $z_{k}$. In other words, $z_{k}$ distributes according to the measure $A_{r_{1}}^{k} \delta_{z_{0}}$, where $\delta_{z_{0}}$ is the Dirac delta-measure at $z_{0}$. Write $z_{k}=x_{k}+y_{k} i$ for $k \in \mathbb{N} \cup\{0\}$.

Recall that in the upper half-plane model, the points at infinity of $\mathbb{H}$ are $\mathbb{R} \cup\{\infty\}$. In the following lemma we show that the random walk $A_{r_{1}}^{k} \delta_{z_{0}}$ moves away from $\infty$ at a constant speed.

Lemma 4.1. Let $f:[0, \pi] \rightarrow[-1,1]$ be the function $f(\theta)=-\frac{1}{r_{1}} \ln \left(e^{r_{1}} \cos ^{2} \theta+e^{-r_{1}} \sin ^{2} \theta\right)$. Let $m$ be the uniform probability measure on $[0, \pi]$ and let $\nu=f^{*} m$ be the induced probability measure on $[-1,1]$ (i.e. for $\left.A \subset[-1,1], \nu(A)=m\left(f^{-1}(A)\right)\right)$. Then $\frac{1}{r_{1}} \ln \left(y_{k}\right)$ distributes according to $\nu * \nu * \ldots * v$ ( $k$ times $)$. In other words, $\ln \left(y_{k}\right)=\ln \left(y_{k-1}\right)+r_{1} Y$, where $Y$ is a random variable, independent from $y_{k-1}$, that distributes according to $\nu$.

Proof. One should show that for a given point $z \in \mathbb{H}$, the logarithm of the imaginary part of the measure $A_{r_{1}} \delta_{z}$ is distributed according to $\ln \left(\operatorname{Im} z^{\prime}\right)=\ln (\operatorname{Im} z)-\ln \left(e^{r_{1}} \cos ^{2} \theta+e^{-r_{1}} \sin ^{2} \theta\right)$, for $0 \leq \theta \leq \pi$ equidistributed.

In the case of $z=i$, the sphere of radius $r_{1}$ around $z$ can be parameterized as

$$
S_{r_{1}}(i)=\left\{\left(\begin{array}{cc}
e^{r_{1} / 2} \sin \theta & e^{-r_{1} / 2} \cos \theta \\
-e^{r_{1} / 2} \cos \theta & e^{-r_{1} / 2} \sin \theta
\end{array}\right) \cdot i=\frac{i+\sin \theta \cos \theta\left(e^{-r_{1}}-e^{r_{1}}\right)}{e^{2 r_{1}} \cos ^{2} \theta+\sin ^{2} \theta} \mid 0 \leq \theta<\pi\right\} .
$$


The logarithm of the imaginary part $y^{\prime}$ of $z^{\prime}=A_{r_{1}} z$ distributes according to $\ln \left(y^{\prime}\right)=-\ln \left(e^{r_{1}} \cos ^{2} \theta+e^{-r_{1}} \sin ^{2} \theta\right)$.

To prove the claim for points other than $z=i$, notice that an isometry $g$ of $\mathbb{H}$ maps $A_{r_{1}} \delta_{z}$ to $A_{r_{1}} \delta_{g \cdot z}$. Since the action of $\left(\begin{array}{ll}1 & s \\ 0 & 1\end{array}\right), s \in \mathbb{R}$ does not change the imaginary coordinate of a point and maps a point $z \in \mathbb{H}$ to $z^{\prime}=z+s$, if the claim holds for $z$, it also holds for $z+s$. Similarly, the action of $\left(\begin{array}{cc}e^{t / 2} & 0 \\ 0 & e^{-t / 2}\end{array}\right)$, $t \in \mathbb{R}$, maps a point $z$ to $z^{\prime}=e^{t} z$, and, in particular, multiplies its imaginary coordinate by $e^{t}$, hence if the claim is true for $z$, it is true for $e^{t} z$ as well. Therefore it is holds for every point $z \in \mathbb{H}$.

Corollary 4.2. The random variables $\sqrt{k}^{-1}\left(k^{-1} r_{1} \ln \left(y_{k}\right)+\alpha_{r_{1}}\right)$ converges in distribution to the normal distribution $N\left(0, \sigma_{r_{1}}^{2}\right)$, where

$$
\begin{aligned}
\alpha_{r_{1}} & =\frac{1}{\pi r_{1}} \int_{0}^{\pi} \ln \left(e^{r_{1}} \cos ^{2} \theta+e^{-r_{1}} \sin ^{2} \theta\right) d \theta, \\
\sigma_{r_{1}}^{2} & =\frac{1}{\pi} \int_{0}^{\pi}\left(\frac{1}{r_{1}} \ln \left(e^{r_{1}} \cos ^{2} \theta+e^{-r_{1}} \sin ^{2} \theta\right)-\alpha_{r_{1}}\right)^{2} d \theta .
\end{aligned}
$$

Also, these numbers satisfies $0<\alpha_{r_{1}}<1$ and that $\sigma_{r_{1}}^{2} \leq 4$.

Moreover, the Hoeffding inequality holds: there exist $c>0$ such that for every $\lambda \geq 0$ and $k \geq 0$

$$
\operatorname{Pr}\left(\left|\ln \left(y_{k}\right)+\alpha_{r} k r_{1}\right| \geq \lambda r_{1} \sqrt{k}\right) \ll e^{-c \lambda^{2}} .
$$

Proof. The statement is a direct application of the central limit theorem and Hoeffding's inequality for independent bounded random variables. The expectancy is equal to $\alpha_{r_{1}}$ and the variance is equal to $\sigma_{r_{1}}$. The fact that $0<\alpha_{r_{1}}<1$ follows from the fact that logarithm is a concave function.

The random walk operator $A_{r_{1}}$ commutes with the action by isometries on $\mathbb{H}$. The stabilizer of $i$ acts transitively on the points at infinity of $\mathbb{H}$. Therefore, just as the random walk $A_{r_{1}}^{k} \delta_{z_{0}}$ moves away from $\infty$, it moves away from any other point at infinity.

Corollary 4.3. Let $g \in G$ be an isometry of $\mathbb{H}$ fixing $i$, then Corollary 4.2 holds if we replace $y_{k}=I m z_{k}$ by $\operatorname{Im}\left(g \cdot z_{k}\right)$, i.e., $\sqrt{k}^{-1}\left(k^{-1} r_{1} \ln \left(\operatorname{Im}\left(g \cdot z_{k}\right)\right)+\alpha_{r_{1}}\right)$ converges in distribution to the normal distribution $N\left(0, \sigma_{r_{1}}^{2}\right)$ with $\alpha_{1}$ and $\sigma_{1}^{2}$ as in Corollary 4.2.

In the following Lemma, we make a particular use of the above Corollary for the isometry $g: z \mapsto-1 / z$.

Lemma 4.4. There exists $c>0$ such that $\operatorname{Pr}\left(x_{k}^{2} \geq \exp \left(\lambda r_{1} k^{1 / 2}\right)\right) \ll e^{-c \lambda^{2}}$ for all $\lambda>0$ and $k \geq 0$.

Proof. By Corollary 4.2 there exists $c_{0}>0$ such that

$$
\operatorname{Pr}\left(\left|\ln \left(y_{k}\right)+\alpha_{r_{1}} k r_{1}\right| \geq \lambda r_{1} \sqrt{k}\right) \ll e^{-c_{0} \lambda^{2}} .
$$

By Corollary 4.3 applied for $-\operatorname{Im} z_{k}^{-1}=\frac{y_{k}}{x_{k}^{2}+y_{k}^{2}}$, there exists $c_{1}>0$ such that

$$
\operatorname{Pr}\left(\left|\ln \left(\frac{y_{k}}{x_{k}^{2}+y_{k}^{2}}\right)+r_{1} \alpha_{r_{1}} k\right| \geq \lambda r_{1} \sqrt{k}\right) \ll e^{-c_{1} \lambda^{2}}
$$

and hence

$$
\operatorname{Pr}\left(\left|\ln \left(x_{k}^{2}+y_{k}^{2}\right)\right| \geq 2 r_{1} \lambda \sqrt{k}\right) \ll e^{-c_{0} \lambda^{2}}+e^{-c_{1} \lambda^{2}} .
$$


Therefore there exists $c>0$ such that

$$
\operatorname{Pr}\left(x_{k}^{2} \geq \exp \left(r_{1} \lambda \sqrt{k}\right)\right) \leq \operatorname{Pr}\left(x_{k}^{2}+y_{k}^{2} \geq \exp \left(r_{1} \lambda \sqrt{k}\right)\right) \ll e^{-c \lambda^{2}} .
$$

Corollary 4.5. Let $z_{k} \propto A_{r_{1}}^{k} \delta_{z_{0}}$. Then there exists $c=c\left(r_{1}\right)>0$, such that for every $k \geq 0$ and $\lambda \geq 0$

$$
\operatorname{Pr}\left(\left|d\left(z_{k}, z_{0}\right)-\alpha_{r_{1}} r_{1} k\right| \geq \lambda \sqrt{k}\right) \ll_{r_{1}} e^{-c \lambda^{2}}
$$

Proof. Let us start by proving that there exists $c>0$, such that for $k \geq 0, \lambda \geq 0$,

$$
\operatorname{Pr}\left(\left|d\left(z_{k}, z_{0}\right)-\alpha_{r_{1}} r_{1} k\right| \geq 1+\lambda r_{1} \sqrt{k}\right) \ll e^{-c \lambda^{2}}
$$

For any point $z=x+i y \in \mathbb{H}$, the triangle inequality implies that

$$
\begin{aligned}
|d(z, i)-d(z, x+i)| & \leq d(x+i, i)=\operatorname{acosh}\left(1+\frac{x^{2}}{2}\right) \\
& \leq \max \left\{1,1+10 \ln \left(x^{2}\right)\right\} .
\end{aligned}
$$

Hence by Lemma 4.4 there exists $c_{0}>0$, such that

$$
\operatorname{Pr}\left(|d(z, i)-d(z, x+i)| \leq 1+\lambda r_{1} \sqrt{k}\right) \ll e^{-c_{0} \lambda^{2}}
$$

And by Corollary 4.2 there exists $c_{1}>0$, such that

$$
\operatorname{Pr}\left(\left|\ln y+\alpha_{r_{1}} k r_{1}\right| \geq \lambda r_{1} \sqrt{k}\right) \ll e^{-c_{1} \lambda^{2}} .
$$

Since $\left|d(z, x+i)-\alpha_{r_{1}} k r_{1}\right|=|| \ln y\left|-\alpha_{r_{1}} k r_{1}\right|$, if || $\ln y\left|-\alpha_{r_{1}} k r_{1}\right| \geq \lambda r_{1} \sqrt{k}$ then also $\left|\ln y+\alpha_{r_{1}} k r_{1}\right| \geq \lambda r_{1} \sqrt{k}$, and

$$
\operatorname{Pr}\left(\left|d(z, x+i)-\alpha_{r_{1}} k r_{1}\right| \geq \lambda r_{1} \sqrt{k}\right) \ll e^{-c_{1} \lambda^{2}},
$$

which completes the proof.

Equation 4.1 follows from Equation 4.2, as for $\lambda \geq r_{1}^{-1}$ and $k>01+\lambda r_{1} \sqrt{k} \leq 2 \lambda r_{1} \sqrt{k}$, and we can choose $c^{\prime}\left(r_{1}\right)=c / r_{1} 2$ and choose the constant of $\ll_{r_{1}}$ in such a way that 4.1 holds for $\lambda \leq r_{1}^{-1}$.

Remark 4.6. One cannot hope to change $\left|d\left(z_{k}, z_{0}\right)-\alpha_{r_{1}} k r_{1}\right| \geq 1+\lambda r_{1} \sqrt{k}$ to $\left|d\left(z_{k}, z_{0}\right)-\alpha_{r_{1}} k r_{1}\right| \geq \lambda r_{1} \sqrt{k}$ in the theorem without assuming dependency on $r_{1}$, since for $r_{1} \rightarrow 0, k \rightarrow \infty$ and $k r_{1} \rightarrow 0$ the random walk behaves like the distance $r_{1}$ random walk in $\mathbb{R}^{2}$, and in particular it will not diverge at a constant speed.

Note that for $f \in L^{2}(\mathbb{H})\left(f \in L^{2}(X)\right.$, resp.), and for $x \in \mathbb{H}(x \in X$, resp. $)$, the following equality holds

$$
A_{r_{1}}^{k} f(x)=\int_{0}^{k r_{1}}\left(A_{r} f\right)(x) d m_{k}^{r_{1}}(r),
$$

for some probability measure $m_{k}^{r_{1}}$ supported on $\left[0, k r_{1}\right]$ and $k \in \mathbb{N}$.

Corollary 4.7. There exists $c=c\left(r_{1}\right)>0$ such that for every $k \geq 0$

$$
\int_{r:\left|r-k r_{1} \alpha_{r_{1}}\right| \leq \lambda r_{1} \sqrt{k}} d m_{k}^{r_{1}}(r) \ll_{r_{1}} \exp \left(-c \lambda^{2}\right) .
$$


Proof. Follows directly from Corollary 4.5.

In the next section we will prove that the measure $m_{k}$ for $k \geq 3$ is actually defined by an $L^{2}$-function $M\left(r_{1}, r\right)$, and $d m_{k}^{r_{1}}(r)=M\left(r_{1}, r\right) d r$.

The Brownian Motion. The Brownian motion is the random walk on $\mathbb{H}$ defined by $B_{t}=\exp (-\Delta t)$. The Brownian motion was studied by many authors, and can be analyzed either by the Helgason-Fourier transform, or by the "distance to infinity" approach used to study the discrete random walk. In any case, based on [4, 2], we may write $B_{t} f(x)=\int p(t, r)\left(A_{r} f\right)(x) d r$, with

$$
p(t, r) \asymp \frac{t^{-1} r}{\sqrt{1+r+t}} \exp \left(-\frac{(r-t)^{2}}{4 t}\right) \ll t^{-1} r \exp \left(-\frac{(r-t)^{2}}{4 t}\right) .
$$

Proposition 4.8. There exist $c>0, t_{0} \geq 0$ such that for every $\lambda>0$ and every $t>t_{0}$

$$
\int_{r:|r-t| \geq \lambda \sqrt{t}} p(t, r) d r \ll_{t_{0}} e^{-c \lambda^{2}}
$$

Proof. We have

$$
\int_{r:|r-t| \geq \lambda \sqrt{t}} p(t, r) d r \leq \int_{-\infty}^{-\lambda} p(t, t+\lambda \sqrt{t}) d \lambda^{\prime}+\int_{\lambda}^{\infty} p\left(t, t+\lambda^{\prime} \sqrt{t}\right) d \lambda^{\prime}
$$

For $r=t-\lambda^{\prime} \sqrt{t} \leq t$, we have

$$
p(t, r) \ll e^{-\frac{\lambda^{\prime 2}}{4}},
$$

so by the standard bound for $\lambda \geq 0$

$$
\int_{-\infty}^{-\lambda} e^{-x^{2}} d x=\int_{-\infty}^{0} e^{-(-\lambda+x)^{2}} d x \leq e^{-\lambda^{2}} \int_{-\infty}^{0} e^{-x^{2}} \ll e^{-\lambda^{2}}
$$

we have

$$
\int_{-\infty}^{-\lambda} p\left(t, t+\lambda^{\prime} \sqrt{t}\right) d \lambda^{\prime} \ll e^{-\frac{\lambda^{2}}{4}}
$$

For $r=t+\lambda^{\prime} \sqrt{t} \geq t$

so for $t \geq t_{0}$

$$
p(t, r) \ll\left(1+\frac{\lambda^{\prime}}{\sqrt{t}}\right) e^{-\frac{\lambda^{\prime 2}}{4}},
$$

$$
\begin{aligned}
\int_{\lambda}^{\infty} p\left(t, t+\lambda^{\prime} \sqrt{t}\right) d \lambda^{\prime} & \ll e^{-\frac{\lambda^{2}}{4}}+\frac{1}{\sqrt{t_{0}}} \int_{\lambda}^{\infty} \lambda^{\prime} e^{-\frac{\lambda^{\prime 2}}{4}} d \lambda^{\prime} \\
& \ll_{t_{0}} e^{-\frac{\lambda^{2}}{4}}+\left.\left(e^{-\frac{\lambda^{\prime 2}}{4}}\right)\right|_{\lambda} ^{\infty} \ll e^{-\frac{\lambda^{2}}{4}}
\end{aligned}
$$




\section{Short Time Bound on the Random Walks}

In this section we show that after a short time both random walks on $X$ can be described by an $L^{2}$-function, whose norm depend is bounded is the injectivity radius of $x_{0}$ is bounded away from 0 .

It was shown in Section 2 that the $L^{2}$-spectrum of the operator $A_{r}$ constitutes of the values of $\varphi_{\frac{1}{2}+i s}\left(e^{r} i\right)$ for $s \in \mathbb{R}$. For the ease of notation we write $\phi(s, r)=\varphi_{\frac{1}{2}+i s}\left(e^{r} i\right)$.

Lemma 5.1. For any $r$, the following inequality holds

$$
|\phi(s, r)| \ll_{r}|s|^{-1 / 2} .
$$

Proof. Up to a constant, the function $\phi(s, r)$ is equal to $\int_{0}^{1} \frac{\cos (s r(1-x))}{\sqrt{\cosh r-\cosh r(1-x)}} d x$. This function is continuous in $s$, hence we may assume that $|s|$ is large enough. Write

$$
\int_{0}^{1} \frac{\cos (s r(1-x))}{\sqrt{\cosh r-\cosh r(1-x)}} d x=\int_{0}^{|s|^{-1}} \frac{\cos (s r(1-x))}{\sqrt{\cosh r-\cosh r(1-x)}} d x+\int_{|s|^{-1}}^{1} \frac{\cos (s r(1-x))}{\sqrt{\cosh r-\cosh r(1-x)}} d x .
$$

Then since $\lim _{x \rightarrow 0^{+}} \frac{\sqrt{x}}{\sqrt{\cosh r-\cosh r(1-x)}}=c_{r}>0$, for $|s|$ large enough we have

$$
\left|\int_{0}^{|s|^{-1}} \frac{\cos (\operatorname{sr}(1-x))}{\sqrt{\cosh r-\cosh r(1-x)}} d x\right| \leq \int_{0}^{|s|^{-1}} \frac{1}{\sqrt{\cosh r-\cosh r(1-x)}} d x \ll_{r} \int_{0}^{|s|^{-1}} \frac{1}{\sqrt{x}} d x \ll \frac{1}{\sqrt{|s|}} .
$$

Analogously,

$$
\int_{|s|^{-1}}^{1} \frac{1}{(\cosh r-\cosh r(1-x))^{3 / 2}} d x \ll_{r} \sqrt{|s|} .
$$

Write $G(x)=-\frac{1}{s r} \sin (s r(1-x)), F(x)=1 / \sqrt{\cosh r-\cosh r(1-x)}$, then by integration by parts,

$$
\begin{aligned}
\int_{|s|^{-1}}^{1} G^{\prime}(x) F(x) d x & =G(1) F(1)-G\left(|s|^{-1}\right) F\left(|s|^{-1}\right)-\int_{|s|^{-1}}^{1} G(x) F^{\prime}(x) d x, \\
& =-G\left(|s|^{-1}\right) F\left(|s|^{-1}\right)-\int_{|s|^{-1}}^{1} G(x) F^{\prime}(x) d x
\end{aligned}
$$

and hence,

$$
\begin{aligned}
\left|\int_{|s|^{-1}}^{1} \frac{\cos (\operatorname{sr}(1-x))}{\sqrt{\cosh r-\cosh r(1-x)}} d x\right| & \ll\left|\frac{1}{s s^{-1}}\right|\left|\frac{1}{\sqrt{\cosh r-\cosh r\left(1-|s|^{-1}\right)}}\right|+\int_{|s|^{-1}}^{1} \frac{1}{|s| r(\cosh r-\cosh r(1-x))^{3 / 2}} d x \\
& \ll_{r}|s|^{-1 / 2}+\frac{1}{|s|} \cdot \sqrt{|s|} \ll \frac{1}{\sqrt{|s|}},
\end{aligned}
$$

which completes the proof.

Lemma 5.2. For any $x_{0} \in \mathbb{H}$, we have $A_{r_{1}}^{3} \delta_{x_{0}} \in L^{2}(\mathbb{H})$. 
Proof. By Theorem 2.1, the Helgason-Fourier transform of $A_{r_{1}}^{3}$ satisfies $\widehat{A_{r_{1}}^{3}(s)}=\left(\widehat{A_{r_{1}}}(s)\right)^{3}=\phi^{3}\left(s, r_{1}\right)$. Applying Lemma 5.1, and using the fact that $\frac{s}{4 \pi} \tanh (\pi s)<s$ implies that

$$
\begin{aligned}
\int_{-\infty}^{\infty}\left|\widehat{A_{r_{1}}^{3}(s)}\right|^{2} \frac{s}{4 \pi} \tanh (\pi s) d s & =\int_{-\infty}^{\infty}\left|\phi\left(s, r_{1}\right)\right|^{6} \frac{s}{4 \pi} \tanh (\pi s) d s \\
& \ll_{r} 1+\int_{|s|>1}|s|^{-3}|s| d s<\infty .
\end{aligned}
$$

Using the inverse Fourier transform we conclude by Corollary 2.2 that $A_{r_{1}}^{3}=\int_{r} f(r) A_{r} d r$, with $f(r)$ an $L^{2}$-function. In particular, $\left\|A_{r_{1}}^{3} \delta_{x_{0}}\right\|_{2}=\int_{r}|f(r)|^{2} d r<\infty$, as needed.

Remark 5.3. For $k=0,1,2$, the analogous statement is not true. For $k=0,1, A_{r_{1}}^{k} \delta_{x_{0}}$ cannot be considered as a function. For $k_{0}=2, A_{r_{1}}^{2}=\int_{0}^{r_{1}} g(r) A_{r} d r, \int_{0}^{r} g\left(r^{\prime}\right) d r^{\prime}=\operatorname{acos}\left(\left(\cosh ^{2}\left(r_{1}\right)-\cosh (r)\right) / \sinh ^{2}\left(r_{1}\right)\right) / \pi$, where $g(r)$ is a function on $X$, but not an $L^{2}$ function.

Lemma 5.4. For $k_{0} \geq 3$ (respectively for $t_{0}>0$ ) there exists a constant $C=C\left(r_{0}, r_{1}, k_{0}\right)$ (resp. $C=$ $\left.C\left(r_{0}, t_{0}\right)\right)$ such that if $x_{0} \in X$ has a injectivity radius at least $r_{0}$ then $A_{r_{1}}^{k_{0}} \delta_{x_{0}} \in L^{2}(X)$ and $\left\|A_{r_{1}}^{k_{0}} \delta_{x_{0}}\right\|_{2} \leq C$ (resp. $B_{t_{0}} \delta_{x_{0}} \in L^{2}(X)$ and $\left\|B_{t_{0}} \delta_{x_{0}}\right\|_{2} \leq C$ ).

Proof. We start with the discrete random walk $A_{r_{1}}^{k_{0}} \delta_{y_{0}}$. Since $\left\|A_{r_{1}}\right\|_{2} \leq 1$ it is enough to assume that $k_{0}=3$.

Let $y_{0} \in \mathbb{H}$ be a fixed point covering $x_{0} \in X$. Let $x_{1} \in X$ be a point different from $x_{0}$. We claim that it has a bounded number $D \ll_{r_{0}, k_{0}, k_{1}} 1$ of points $z_{1}, \ldots, z_{D} \in B_{k_{0} r_{0}}\left(y_{0}\right)$ covering $x_{1}$. Since $A_{r_{1}}^{k_{0}} \delta_{y_{0}} \in L^{2}(\mathbb{H})$, it is supported on $B_{k_{0} r_{0}}\left(y_{0}\right)$ and $A_{r_{1}}^{k_{0}} \delta_{x_{0}}$ is the push-forward of $A_{r_{1}}^{k_{0}} \delta_{y_{0}}$ to $X$, this claim will give the lemma for the discrete random walk. We may assume that $d_{0}=d\left(x_{0}, x_{1}\right)<k_{0} r_{1}$. Let therefore $z_{1}, z_{2}, . . \in B_{k_{0} r_{1}}\left(y_{0}\right)$ be a sequence of different points covering $x_{1}$. Then each such point $z_{i} \in \mathbb{H}$ can be associated with another point $y_{i} \in \mathbb{H}$, covering $x_{0}$, with $d\left(y_{i}, z_{i}\right)=d_{0}$. Moreover, we may choose $y_{i}$ such that $y_{i} \neq y_{j}$ for $z_{i} \neq z_{j}$. By the injectivity radius assumption, $d\left(y_{i}, y_{j}\right) \geq 2 r_{0}$ for $i \neq j$. All the $y_{i}$ 's are contained in the ball $B_{2 k_{0} r_{1}}\left(y_{0}\right)$, and their number is therefore bounded by $\frac{\mu\left(B_{2 k_{0} r_{1}}\right)}{\mu\left(B_{r_{0}}\right)} \ll_{r_{0}, k_{0}, k_{1}} 1$.

Now we turn to the Brownian motion. Since $\left\|B_{t}\right\|_{2} \leq 1$ and $B_{t+t^{\prime}}=B_{t} B_{t^{\prime}}$ we may assume that $t_{0}$ is small enough so that $p_{2}\left(r, t_{0}\right)$ is decreasing for $r>r_{0}$ and $B_{t_{0}} \delta_{y_{0}}(z) \leq e^{-c d\left(y_{0}, z\right)^{2}}$ for some $c=c\left(r_{0}, t_{0}\right)>0$ and $d\left(y_{0}, z\right)>r_{0}$.

Let $y_{0} \in \mathbb{H}$ be again a fixed point covering $x_{0} \in X$. Let $x_{1} \in X$ be another point and let $d_{1}=d\left(x_{0}, x_{1}\right)$. Each point $z_{i}$ covering $x_{1}$ satisfies $d\left(y_{0}, z_{i}\right) \geq d_{1}$ and the number of points $z_{i}$ covering $x_{1}$ of distance $d\left(y_{0}, z_{i}\right) \leq r$ is at most $D_{r} \leq \frac{\mu\left(B_{r+d_{1}}\right)}{\mu\left(B_{r_{0}}\right)} \ll_{r_{0}} e^{d_{1}+r}$. Therefore we get the bound:

$$
\begin{aligned}
B_{t_{0}} \delta_{x_{0}}(x) & =\sum_{z_{i}} B_{t_{0}} \delta_{y_{0}}\left(z_{i}\right)=\sum_{k=0}^{\infty} \sum_{z_{i}: d_{1}+r_{0} k \leq d\left(y_{0}, z_{i}\right) \leq d_{1}+r_{0}(k+1)} B_{t_{0}} \delta_{y_{0}}\left(z_{i}\right) \\
& \leq \sum_{k=0}^{\infty} D_{d_{1}+r_{0}(k+1)} \cdot e^{-c\left(d_{1}+r_{0} k\right)^{2}} \ll_{r_{0}} \sum_{k=0}^{\infty} e^{d_{1}+d_{1}+r_{0}(k+1)-c\left(d_{1}+r_{0} k\right)^{2}} \\
& \ll r_{0}, t_{0} e^{2 d_{1}-c^{\prime} d_{1}^{2}} .
\end{aligned}
$$


For some constant $c^{\prime}>0$ depending on $r_{0}, t_{0}$. Finally, using the fact that the volume of $x \in X$ with $d\left(x_{0}, x\right) \leq d_{1}$ is $\ll e^{d_{1}}$,

$$
\left\|B_{t_{0}} \delta_{x_{0}}\right\|_{2}^{2}=\int_{X}\left|B_{t_{0}} \delta_{x_{0}}(x)\right|^{2} d x \ll_{t_{0}, r_{0}} \int_{d_{1} \geq 0} e^{2\left(2 d_{1}-c^{\prime} d_{1}^{2}\right)} \cdot e^{d_{1}} d t \ll_{t_{0}, r_{0}} 1,
$$

and the lemma is proved.

\section{Proof of Theorem 1.2}

Proof. of Theorem 1.2. We prove the claim for the discrete random walk only. The proof for the Brownian motion is analogous, and exploits Corollary 4.8 instead of Corollary 4.7 and the Brownian motion part of Lemma 5.4 instead of its discrete part.

Suppose $r k \alpha<R_{X}-\lambda \sqrt{R_{X}}$. Let $Y=\left\{y \in X: d\left(x_{0}, y\right)>R_{X}-\frac{\lambda}{2} \sqrt{R_{X}}\right\}$. As $R_{X} \rightarrow \infty$,

$$
1 \geq \mu(Y) / \mu(X) \geq\left(\mu(X)-\mu\left(B\left(R_{X}-\frac{\lambda}{2} \sqrt{R_{X}}\right)\right)\right) / \mu(X) \rightarrow 1,
$$

so $\mu(Y) / \mu(X) \rightarrow 1$.

By Corollary 4.7 , there exists $c_{1}\left(r_{1}\right), C_{1}\left(r_{1}\right)$ such that for $R_{X}$ large enough,

$$
\begin{gathered}
\int_{Y}\left|A_{r}^{k} \delta_{x_{0}}(x)\right| d \mu<C_{1} e^{-c_{1} \lambda^{2}} \\
\int_{X-Y}\left|A_{r}^{k} \delta_{x_{0}}(x)\right| d \mu>1-C_{1} e^{-c_{1} \lambda^{2}} .
\end{gathered}
$$

Therefore,

$$
\begin{aligned}
\left\|A_{r}^{k} b_{x_{0}, r_{0}}-\pi\right\|_{1} & =\int_{Y}\left|A_{r}^{k} \delta_{x_{0}}(x)-\pi(x)\right| d \mu+\int_{X-Y}\left|A_{r}^{k} \delta_{x_{0}}(x)-\pi(x)\right| d \mu \\
& \geq \int_{Y}|\pi(x)| d \mu-\int_{Y}\left|A_{r}^{k_{n}} \delta_{x_{0}}(x)\right| d \mu+\int_{X-Y}\left|A_{r}^{k} \delta_{x_{0}}(x)\right|-\int_{X-Y}|\pi(x)| d \mu \\
& \geq \mu(X)^{-1} \mu(Y)-C_{1} e^{-c_{1} \lambda^{2}}+1-C_{1} e^{-c_{1} \lambda^{2}}-\mu(X)^{-1} \mu(X-Y) \\
& =2 \mu(X)^{-1} \mu(Y)-2 C_{1} e^{-c_{1} \lambda^{2}}
\end{aligned}
$$

and the first bound follows by letting $R_{X} \rightarrow \infty$. Notice that it does not require the Ramanujan assumption.

For the second bound, recall that we may write $A_{r_{1}}^{k} b_{x_{0}, r_{0}}(x)=\int_{r}\left(A_{r} b_{x_{0}, r_{0}}\right)(x) d m_{k}(r)$. Assume that $k r_{1} \alpha>R_{X}+\lambda \sqrt{R_{X}}$. By Corollary 4.7, for some $c_{2}\left(r_{1}\right)>0$, for $R_{X}$ large enough (depending on $r_{1}, \lambda$ ),

$$
\int_{r<R_{X}+\frac{\lambda}{2} \sqrt{R_{X}}} d m_{k} r \ll_{r_{1}} e^{-c_{2} \lambda^{2}} .
$$

As $x_{0} \in X$ has an injectivity radius at least $r_{0}$, by Lemma 5.4, there exists a constant $C_{3}=C\left(r_{0}, r_{1}\right)$ such that $\left\|A_{r_{1}}^{3} \delta_{x_{0}}\right\|_{2} \leq C_{3}$. 
For every $f \in L_{2}(X)$, Cauchy-Schwartz inequality implies that $\|f\|_{1} \leq \sqrt{\mu(X)}\|f\|_{2}$. Therefore,

$$
\begin{aligned}
\left\|A_{r_{1}}^{k} f-\pi\right\|_{1} \leq & \int_{r}\left\|A_{r} \delta_{x_{0}}-\pi\right\|_{1} d m_{k} r= \\
& =\int_{r<R_{X}+\frac{\lambda}{2} \sqrt{R_{X}}}\left\|A_{r} b_{x_{0}, r_{0}}-\pi\right\|_{1} d m_{k} r+\int_{r \geq R_{X}+\frac{\lambda}{2} \sqrt{R_{X}}}\left\|A_{r} b_{x_{0}, r_{0}}-\pi\right\|_{1} d m_{k} r \\
& \leq \int_{r<R_{X}+\frac{\lambda}{2} \sqrt{R_{X}}} 2 d m_{k} r+\int_{r \geq R_{X}+\frac{\lambda}{2} \sqrt{R_{X}}} \mu(X)^{1 / 2}\left\|A_{r}\left(b_{x_{0}, r_{0}}-\pi\right)\right\|_{2} d m_{k} r \\
& \ll r_{1} e^{-c_{2} \lambda^{2}}+\int_{r \geq R_{X}+\frac{\lambda}{2} \sqrt{R_{X}}} \mu(X)^{1 / 2}(r+1) e^{-r / 2} d m_{k} r \\
& \ll e^{-c_{2} \lambda^{2}}+\mu(X)^{1 / 2}\left(R_{X}+\frac{\lambda}{2} \sqrt{R_{X}}+1\right) e^{-\frac{1}{2}\left(R_{X}+\frac{\lambda}{2} \sqrt{R_{X}}\right)} \\
& \ll e^{-c_{2} \lambda^{2}}+\mu(X)^{1 / 2} e^{-\frac{1}{2} R_{X}}\left(R_{X}+\frac{\lambda}{2} \sqrt{R_{X}}+1\right) e^{-\frac{1}{4} \sqrt{R_{X}}} \\
& \rightarrow{ }_{R_{X} \rightarrow \infty} e^{-c_{2} \lambda^{2}},
\end{aligned}
$$

and the second bound follows.

Remark 6.1. The theorem holds for $\lambda>0$ such that $R_{X} \gg_{r_{0}, r_{1}, \lambda} 1$. In other words, $R_{X}$ has to be larger than some constant $R\left(r_{0}, r_{1}, \lambda\right)$ that depends on $r_{0}, r_{1}$ and $\lambda$. By fixing $r_{0}, r_{1}$, one can find the relation between this constant and $\lambda$, namely, it should hold that $\lambda=o\left(\sqrt{R\left(r_{0}, r_{1}, \lambda\right)}\right)$ and $\ln \left(R\left(r_{0}, r_{1}, \lambda\right)\right)=o\left(\lambda \sqrt{R\left(r_{0}, r_{1}, \lambda\right)}\right)$.

\section{7. $L^{p}$-Bounds}

The above results assume $X$ to be Ramanujan. However, similar results can be proved in a general setting. In this section and the next one, we discuss Theorem 1.1 only, but similarly one can elaborate on Theorem 1.2 as well.

The following lemma is well known (see [18]):

Lemma 7.1. The spectrum of the $\Delta$ on $L_{0}^{2}(X)$ below $1 / 4$ is discrete, and corresponds to a finite number of eigenvalues with multiplicities.

Eigenvalues of $\Delta$ strictly below $1 / 4$ are called exceptional. A nice way to measure how far is a representation $V$ of $G$ from being tempered is to ask what is the minimal $p \geq 2$ such that the $K$-finite matrix coefficients of $G$ on $V$ lie in $L^{p}(G)$. The following proposition relates this property to the spectra of the Laplacian and of the operators $A_{r}$. See [17] for the corresponding result on graphs.

Proposition 7.2. The following are equivalent for $p \geq 2$ :

(1) For every $r \geq 0$, the norm of $A_{r}$ on $V$ is bounded by $(r+1) e^{-r / p}$.

(2) Every matrix coefficient of a subrepresentation of $G$ on $L_{0}^{2}(\Gamma \backslash G)$ with $K=P S O_{2}(\mathbb{R})$ fixed vectors is in $L^{p+\epsilon}(G)$ for every $\epsilon>0$.

(3) The spectrum of $\Delta$ on $L_{0}^{2}(X)$ is bounded from below by $\frac{1}{4}-\left(\frac{1}{2}-p^{-1}\right)^{2}$.

The equivalence is also true for every $\Delta$-invariant closed subspace $V \subset L_{0}^{2}(X)$, where in (2) we look at the $G$-subrepresentation generated by $V$. 
Proof. Let $V$ be a $\Delta$-invariant closed subspace of $L_{0}^{2}(X)$.

The complementary series is determined by a real parameter $t, 0 \leq t \leq 1$, with corresponding spherical function $\varphi_{t}$. Write $t=\frac{1}{2}+s_{t}^{\prime}$ and $p_{t}=\left(\frac{1}{2}-\left|s_{t}^{\prime}\right|\right)^{-1}$ (with the convention that $0^{-1}=\infty$ ). The corresponding eigenvalue of the $\Delta$ on $\varphi_{t}$ is

$$
\lambda_{t}=t(1-t)=\frac{1}{4}-s_{t}^{\prime 2}=\frac{1}{4}-\left(\frac{1}{2}-p_{t}^{-1}\right)^{2} .
$$

The eigenvalue of $A_{r}$ on $\varphi_{t}$ is:

$$
\varphi_{t}(r)=\frac{1}{\sqrt{2} \pi} r \int_{-1}^{1} \frac{\exp \left(s_{t}^{\prime} r x\right)}{\sqrt{\cosh r-\cosh r x}} d x .
$$

Recall that for $r>0, \cosh r-\cosh (r x) \geq(\cosh r-1)\left(1-x^{2}\right)$ holds. Hence

$$
\begin{aligned}
\left|\varphi_{t}(r)\right| & =\frac{1}{\pi \sqrt{2}} r\left|\int_{-1}^{1} \frac{\exp \left(s_{t}^{\prime} r x\right)}{\sqrt{\cosh r-\cosh r x}} d x\right| \leq \frac{1}{\pi \sqrt{2}} r \frac{1}{\sqrt{\cosh r-1}} \int_{-1}^{1} \frac{\exp \left(s_{t}^{\prime} r x\right)}{\sqrt{1-x^{2}}} d x \\
& \leq \frac{1}{\sqrt{2} \pi} r \frac{\exp \left(\left|s_{t}^{\prime}\right| r\right)}{\sqrt{\cosh r-1}} \int_{-1}^{1} \frac{1}{\sqrt{1-x^{2}}} d x=\frac{1}{\sqrt{2}} r(\cosh r-1)^{-1 / 2} \exp \left(\left|s_{t}^{\prime}\right| r\right) \\
& \leq(r+1) e^{-r\left(\frac{1}{2}-\left|s_{t}^{\prime}\right|\right)}=(r+1) e^{-r / p_{t}}
\end{aligned}
$$

This proves an implication from (3) to (1).

We also want to give a lower bound on $\left|\varphi_{t}(r)\right|$. Let $f(x)=\cosh r-\cosh (r(1-x))$. For $x \geq 0$, by the Taylor series $f(x)=x r \sinh r-\frac{1}{2} r^{2} x^{2} \cosh \left(r\left(1-x^{\prime}\right)\right), 0 \leq x^{\prime} \leq x$, so $f(x) \leq x r \sinh r$. So for a fixed $\epsilon>0$, and $r \geq 1$,

$$
\begin{aligned}
\varphi_{t}(r) & =\frac{1}{\sqrt{2} \pi} r \int_{-1}^{1} \frac{\exp \left(s_{t}^{\prime} r x\right)}{\sqrt{\cosh r-\cosh r x}} d x \gg r \int_{0}^{2} \frac{\exp \left(s_{t}^{\prime} r(1-x)\right)}{\sqrt{\cosh r-\cosh r(1-x)}} d x \\
& \geq r \int_{0}^{\epsilon} \frac{\exp \left(s_{t}^{\prime} r(1-x)\right)}{\sqrt{\cosh r-\cosh r(1-x)}} d x \\
& \geq \frac{\sqrt{r} e^{s_{t}^{\prime} r}}{\sqrt{\sinh r}} \int_{0}^{\epsilon} \frac{\exp \left(-s_{t}^{\prime} r x\right)}{\sqrt{x}} d x \gg \frac{e^{s_{t}^{\prime} r(1-\epsilon)}}{\sqrt{\sinh r}} \sqrt{\epsilon} \gg \sqrt{\epsilon} e^{-r\left(\frac{1}{2}-\left|s_{t}^{\prime}\right|(1-\epsilon)\right) .}
\end{aligned}
$$

This implies that if for every $r \geq 0, \varphi_{t}(r) \leq(r+1) e^{-r\left(\frac{1}{2}-S\right)}$ then $\left|s_{t}^{\prime}\right| \leq S$. This proves the implication from (1) to (3).

Arguing as in Proposition 2.5, we see that (2) is equivalent to:

- For every $f, f^{\prime} \in V$ and for every $\epsilon>0$,

$$
\int_{r \geq 0} e^{r}\left|\left\langle f, A_{r} f^{\prime}\right\rangle\right|^{p+\epsilon} d r<\infty
$$

We can immediately see that as in Proposition 2.5, this proves the implication from (1) to (2). 
Assume now that (1) and (3) do not hold for $p=p_{0} \geq 2$. By Lemma 7.1, there is an eigenvector $f \in V$ which satisfies $\left\langle f, A_{r} f\right\rangle=\varphi_{t}(r)$, for some $\varphi_{t}$, with $p_{t}>p_{0}$. By Equation 7.1, for some $\delta>0$ and for $r$ large enough $\left|\left\langle f, A_{r} f\right\rangle\right| \gg_{\delta} e^{-r\left(1 / p_{0}+\delta\right)}$. Then Equation 7.2 does not hold, and (2) does not hold.

By Lemma 7.1, for each $X$ there is a minimal $p_{0}$ satisfying the equivalent conditions of Proposition 7.2. Denote it by $p_{0}(X)$. For example, Selberg's lower bound 3/16 implies that for each $X$ corresponding to a congruence subgroup of $S L_{2}(\mathbb{Z}), p_{0}(X) \leq 4$. Further improvements (see e.g. [26]) improve this bound as well. Without any further information about $X$, we can say the following:

Theorem 7.3. Let $r_{0}>0$ be fixed. Let $p=p_{0}(X)$ and assume $R_{X} \geq 1$. Let $x_{0} \in X$ be a point with injectivity radius at least $r_{0}$. Then for every $\gamma>0$

$$
\mu_{X}\left(x \in X: d_{X}\left(x, x_{0}\right) \geq \frac{p}{2}\left(R_{X}+\gamma \ln \left(R_{X}\right)\right)\right) / \mu(X) \ll_{p, r_{0}}\left(1+\gamma^{2}\right) R_{X}^{2-\gamma} .
$$

Proof. The proof is essentially the same as the proof of Theorem 1.1, and we only write the differences. Instead of choosing $r^{\prime}=R_{X}+\gamma \ln \left(R_{X}\right)-r_{0}$, choose $r^{\prime}=\frac{p}{2}\left(R_{X}+\gamma \ln \left(R_{X}\right)\right)-r_{0}$. Then:

$$
\begin{aligned}
\left\|A_{r^{\prime}} b_{x_{0}, r_{0}}-\pi\right\|_{2} & =\left\|A_{r^{\prime}}\left(b_{x_{0}, r_{0}}-\pi\right)\right\|_{2} \leq\left(r^{\prime}+1\right) e^{-r^{\prime} / p}\left\|b_{x_{0}, r_{0}}-\pi\right\|_{2} \\
& \ll_{r_{0}}\left(r^{\prime}+1\right) e^{-\frac{1}{2} R_{X}-\frac{1}{2} \gamma \ln \left(R_{X}\right)+r_{0} / p} \\
& \ll_{r_{0}, p} \frac{\frac{p}{2}\left(R_{X}+\gamma \ln \left(R_{X}\right)\right)-r_{0}}{R_{X}} \mu(X)^{-1 / 2} e^{-\frac{1}{2}(\gamma-2) \ln \left(R_{X}\right)} \\
& \ll_{r_{0}, p}(1+\gamma) \mu(X)^{-1 / 2} e^{-\frac{1}{2}(\gamma-2) \ln \left(R_{X}\right)} .
\end{aligned}
$$

The rest of the proof is the same.

\section{Covers}

Let $X_{0}=\Gamma_{0} \backslash \mathbb{H}$. Then a finite index subgroup $\Gamma_{X} \subset \Gamma_{0}$ defines a cover $X=\Gamma_{X} \backslash \mathbb{H}$ of $X_{0}$, with cover map $\rho: X \rightarrow X_{0}$. The pull-back $\rho^{*}: L^{2}\left(X_{0}\right) \rightarrow L^{2}(X)$ defines a closed subspace $\rho^{*} L^{2}\left(X_{0}\right) \subset L^{2}(X)$. Denote the orthogonal complement of $\rho^{*} L^{2}\left(X_{0}\right)$ in $L^{2}(X)$ by $L^{2}\left(X / X_{0}\right)$.

For $p>2$, denote by $m(X, p)$ the dimension of the space spanned by eigenvectors of $L^{2}\left(X / X_{0}\right)$ whose matrix coefficients are not in $L^{p^{\prime}}$ for every $p^{\prime}<p$ but are in $L^{p^{\prime}}$ for $p^{\prime}>p$. Denote also $M(X, p)=$ $\sum_{p^{\prime} \geq p} m\left(X, p^{\prime}\right)$.

A cover $\rho: X \rightarrow X_{0}$ is called normal if $\Gamma_{X} \subset \Gamma_{X_{0}}$ is a normal subgroup. Equivalently, a cover $\rho: X \rightarrow X_{0}$ is normal if there exists a group $H$ acting on $X$ such that $\rho(x)=\rho(y)$ if and only if $x$ and $y$ are on the same $H$-orbit. We call $H$ the cover group.

Our main result about covers is as the following theorem. Note that if $X$ is an $N$-cover of $X_{0}$ then $\mu(X)=N \cdot \mu\left(X_{0}\right)$. Therefore, $\mu(X) \asymp X_{0} N$ and $R_{X}=\ln (N)+O_{X_{0}}(1)$.

Theorem 8.1. Let $r_{0}>0$ be fixed, and let $X_{0}$ be a fixed quotient. Let $\rho_{q}: X_{q} \rightarrow X_{0}$ be family of normal $N_{q}$-covers, with $N_{q} \rightarrow \infty$ as $q \rightarrow \infty$.

Assume that $g: \mathbb{R}_{+} \rightarrow \mathbb{R}_{+}$is non-decreasing function satisfying:

(1) For some fixed $\delta>2$ and for $R$ large enough, $g(R) \geq R+\delta \ln R$. 
(2) Either

or

$$
g^{3}\left(\ln \left(N_{q}\right)\right) \sum_{p: m\left(X_{q}, p\right) \neq 0} e^{-2 g\left(\ln \left(N_{q}\right)\right) / p_{i}} m\left(X_{q}, p_{i}\right)=o(1)
$$

$$
\begin{gathered}
g^{3}\left(\ln \left(N_{q}\right)\right) \int_{2}^{\infty} M\left(X_{q}, p\right) e^{-2 g\left(\ln \left(N_{q}\right)\right) / p} p^{-2} d p=o(1), \text { and } \\
g^{2}\left(\ln \left(N_{q}\right)\right) \lim _{p \rightarrow 2, p>2} M\left(X_{q}, p\right) e^{-g\left(\ln \left(N_{q}\right)\right)}=o(1)
\end{gathered}
$$

For every $q$, let $x_{0}^{(q)} \in X_{q}$ be a point such that its projection $\rho_{q}\left(x_{0}^{(q)}\right)$ to $X_{0}$ has injectivity radius at least $r_{0}$. Then

$$
\mu\left(x \in X_{q}: d_{X_{q}}\left(x, x_{0}^{(q)}\right) \geq g\left(\ln \left(N_{q}\right)\right)\right) / \mu\left(X_{q}\right)=o(1),
$$

where the implied constant depends on $X_{0},\left\{X_{q}\right\}, r_{0}$ and $g$.

Before proving the theorem, let us study its corollaries.

Definition 8.2. We say that a family of covers $\left\{X_{q}\right\}$ of $X_{0}$ satisfies a density condition with parameter $A$ if for every $\epsilon>0$, for each $p>2$,

$$
M(X, p) \ll_{\epsilon,\left\{X_{q}\right\}, X_{0}} C N^{1-A(p-2) / p+\epsilon},
$$

and furthermore

- The number of exceptional eigenvalues $\lim _{p \rightarrow 2, p>2} M(X, p)=\sum_{p>2} m(X, p)$ of $X_{q}$ is $\ll_{\left\{X_{q}\right\}, X_{0}} N$.

- There exists $p_{\max }$ such that $M\left(X, p_{\max }\right)=0$.

The assumption that the number of exceptional eigenvalues is $O(N)$ is well known to hold in the arithmetic case (see [25]). There are two main instances of such density results:

(1) The case $A=1$ : in this case we may simply write $M(X, p) \ll_{\epsilon, X_{0}} N^{2 / p+\epsilon}$. This is known to hold for a wide range of cases, including the congruence subgroups of $S L_{2}(\mathbb{Z})$ and all cocompact arithmetic lattices in $S L_{2}(\mathbb{R})$ (See [25, 27] for the uniform case and [13] for $S L_{2}(\mathbb{Z})$ ). The corresponding result for LPS graphs are implicitly contained in [3, Section 4.4]. In this case, for prime congruence, one may find $p_{\max }$ by using lower bounds on the dimensions of representations of $S L_{2}\left(F_{q}\right)$ (See [27]).

(2) The case $A>1$ : this case requires deeper results in analytic number theory, and applies to congruence subgroups of $S L_{2}(\mathbb{Z})$. In this case, $p_{\max }$ is essentially bounded by $\frac{2}{1-A^{-1}}$, and there is no need to restrict to prime congruence. See [15], and [12] and the references therein for recent results .

Corollary 8.3. Let $\rho: X_{q} \rightarrow X_{0}$ be family of normal $N_{q}$-covers, with $N_{q} \rightarrow \infty$. Assume the family satisfies a density condition with parameter $A \geq 1$.

Let $x_{0}^{(q)} \in X_{q}$ be a point such that its projection $\rho_{q}\left(x_{0}^{(q)}\right)$ to $X_{0}$ has injectivity radius at least $r_{0}$. Then for every $\epsilon_{0}>0$

$$
\mu\left(x \in X_{q}: d_{X_{q}}\left(x, x_{0}^{(q)}\right) \geq R_{X_{q}}\left(1+\epsilon_{0}\right)\right) / \mu\left(X_{q}\right)=o(1) .
$$

Proof. One should verify Inequalities 8.2,8.3. We may assume $A=1$. 
Let $g(R)=\left(1+\epsilon_{0}\right) R$, then for $\epsilon>0$ small enough with respect to $\epsilon_{0}$ it holds that

$$
\begin{aligned}
& g^{3}\left(\ln \left(N_{q}\right)\right) \int_{2}^{\infty} M(X, p) e^{-2 g\left(\ln \left(N_{q}\right)\right) / p} p^{-2} d p \\
& \ll_{\epsilon_{0} \cdot\left\{X_{q}\right\}, X_{0}}\left(1+e_{0}\right)^{3} \ln ^{3}\left(N_{q}\right) \int_{2}^{p_{\max }} N_{q}^{2 / p+\epsilon} e^{-2\left(1+\epsilon_{0}\right) \ln \left(N_{q}\right) / p} p^{-2} d p \\
& \ll_{\epsilon_{0} \cdot\left\{X_{q}\right\}, X_{0}} \ln ^{3}\left(N_{q}\right) \int_{2}^{p_{\max }} N_{q}^{2 / p+\epsilon} N_{q}^{-2\left(1+\epsilon_{0}\right) / p} p^{-2} d p \\
& \ll_{X_{0}} \ln ^{3}\left(N_{q}\right) \int_{2}^{p_{\max }} N_{q}^{\epsilon-2 \epsilon_{0} / p_{\max }} p^{-2} d p \\
& \ll \ln ^{3}\left(N_{q}\right) N_{q}^{\epsilon-2 / p_{\max } \epsilon_{0}} \rightarrow_{N_{q} \rightarrow \infty} 0 .
\end{aligned}
$$

In addition,

$$
\begin{aligned}
& g^{2}\left(\ln \left(N_{q}\right)\right) \lim _{p \rightarrow 2, p>2} M\left(X_{q}, p\right) e^{-g\left(\ln \left(N_{q}\right)\right)} \\
& \ll_{\left\{X_{q}\right\}, X_{0}}\left(1+\epsilon_{0}\right)^{2} \ln ^{2}\left(N_{q}\right) N_{q}^{1-\left(1+\epsilon_{0}\right)} \\
& \ll_{\epsilon_{0}} \ln ^{2}\left(N_{q}\right) N_{q}^{\epsilon_{0}} \rightarrow_{N_{q} \rightarrow \infty} 0
\end{aligned}
$$

Remark 8.4. The density theorems with parameter $A>1$ are not far from proving that there exists $C>0$ such that $\mu\left(x \in X_{q}: d_{X_{q}}\left(x, x_{0}^{(q)}\right) \geq R_{X_{q}}+C \ln R_{X_{q}}\right) / \mu\left(X_{q}\right)=o(1)$. The required bound is that for some $\epsilon_{2}>0, C_{2}>0$ and every $2<p<p+\epsilon_{0}, \mathrm{M}\left(X_{q}, p\right) \ll \ln ^{C_{2}}(N) \cdot N^{2 / p}$.

Let us turn to the proof of Theorem 8.1. It will depend on the following two Lemmas.

Lemma 8.5. Let $\rho: X \rightarrow X_{0}$ be an $N$-cover, $U=\rho^{*} L_{0}^{2}\left(X^{\prime}\right) \subset L_{0}^{2}(X)$ be the space of functions pulled back from $X_{0}$ to $X$ and let $P_{U}$ be the orthogonal projection onto $U$. Let $x_{0} \in X$ be a point such that its projection to $X_{0}$ has injectivity radius at least $r_{0}$. Then

$$
\left\|P_{U}\left(b_{x_{0}, r_{0}}\right)\right\|_{2}=N^{-1 / 2}\left\|b_{x_{0}, r_{0}}\right\|_{2} .
$$

Proof. We have

$$
\left\|P_{U}\left(b_{x_{0}, r_{0}}\right)\right\|_{2}=\max _{u \in U,\|u\|_{2}=1}\left\langle u, b_{x_{0}, r_{0}}\right\rangle=\max _{u^{\prime} \in L^{2}\left(x^{\prime}\right),\left\|\rho^{*} u^{\prime}\right\|_{2}=1}\left\langle\rho^{*} u^{\prime}, b_{x_{0}, r_{0}}\right\rangle .
$$

But $\left\|\rho^{*} u^{\prime}\right\|_{2}^{2}=N\left\|u^{\prime}\right\|_{2}^{2}$ and $\left\langle\rho^{*} u^{\prime}, b_{x_{0}, r_{0}}\right\rangle=\left\langle u^{\prime}, b_{\rho\left(x_{0}\right), r_{0}}\right\rangle$. So

$$
\left\|P_{U}\left(b_{x_{0}, r_{0}}\right)\right\|_{2}=\max _{u^{\prime} \in L^{2}\left(x^{\prime}\right),\left\|u^{\prime}\right\|_{2}=N^{-1 / 2}}\left\langle u^{\prime}, b_{\rho\left(x_{0}\right), r_{0}}\right\rangle=N^{-1 / 2}\left\|b_{\rho\left(x_{0}\right), r_{0}}\right\|_{2}=N^{-1 / 2}\left\|b_{x_{0}, r_{0}}\right\|_{2} .
$$

Lemma 8.6. Let $\rho: X \rightarrow X_{0}$ be a normal $N$-cover, with cover group $H$. Let $W \subset L^{2}(X)$ be a finite dimensional $H$-invariant subspace and $P_{W}$ the orthogonal projection onto this subspace. Let $x_{0} \in X$ be a 
point such that its projection to $X_{0}$ has injectivity radius at least $r_{0}$. Then

$$
\left\|P_{W}\left(b_{x_{0}, r_{0}}\right)\right\|_{2} \leq \sqrt{\frac{\operatorname{dim} W}{N}}\left\|b_{x_{0}, r_{0}}\right\|_{2} .
$$

Proof. Let $u_{1}, \ldots, u_{\operatorname{dim} W}$ be an orthonormal basis of $W$. Then

$$
\left\|P_{W}\left(b_{x_{0}, r_{0}}\right)\right\|_{2}^{2}=\sum_{i=1}^{\operatorname{dim} W}\left|\left\langle u_{i}, b_{x_{0}, r_{0}}\right\rangle\right|^{2} .
$$

On the other hand, the points $h x_{0}$, where $h \in H$, are all distinct, the balls $B_{r}\left(h x_{0}\right)$ of radius $r_{0}$ around them are disjoint, and since $W$ is $H$-invariant for each $h \in H$

$$
\left\|P_{W}\left(b_{h x_{0}, r_{0}}\right)\right\|_{2}^{2}=\left\|P_{W}\left(b_{x_{0}, r_{0}}\right)\right\|_{2}^{2} .
$$

so

$$
\begin{aligned}
N\left\|P_{W}\left(b_{x_{0}, r_{0}}\right)\right\|_{2}^{2} & =\sum_{h \in H} \sum_{i=1}^{\operatorname{dim} W}\left|\left\langle u_{i}, b_{h x_{0}, r_{0}}\right\rangle\right|^{2} \\
& \leq \sum_{i=1}^{\operatorname{dim} W} \sum_{h \in H}\left\|\left.u_{i}\right|_{B_{r}\left(h x_{0}\right)}\right\|_{2}^{2}\left\|b_{x_{0}, r_{0}}\right\|_{2}^{2} \\
& =\left\|b_{x_{0}, r_{0}}\right\|_{2}^{2} \sum_{i=1}^{\operatorname{dim} W} \sum_{h \in H}\left\|\left.u_{i}\right|_{B_{r}\left(h x_{0}\right)}\right\|_{2}^{2} \\
& \leq\left\|b_{x_{0}, r_{0}}\right\|_{2}^{2} \sum_{i=1}^{\operatorname{dim} W}\left\|u_{i}\right\|_{2}^{2}=\operatorname{dim} W\left\|b_{x_{0}, r_{0}}\right\|_{2}^{2} .
\end{aligned}
$$

Proof. of Theorem 8.1. To avoid cumbersome notations we do not use the index $q$ in the proof below.

By the proof of Theorem 1.1 one should prove the following inequality for $r=g\left(R_{X}\right)$,

$$
\left\|A_{r}\left(b_{x_{0}, r_{0}}-\pi\right)\right\|_{2}^{2}=o\left(N^{-1}\right) .
$$

Let $\left\{p_{i}\right\}_{i=1}^{T}$ be the set of $p$-values (without multiplicities) of exceptional eigenvalues of $L^{2}\left(X / X_{0}\right)$, i.e., the $p$ such that the corresponding matrix coefficient is not in $L^{p^{\prime}}$ for every $p^{\prime}<p$ but are in $L^{p^{\prime}}$ for every $p^{\prime}>p$. Let $V_{i}$ be the vector space of eigenvectors with $p$-value $p_{i}$. Let $p_{0}=2$ and $V_{0}$ the orthogonal complement of the $V_{i}$ in $L^{2}\left(X / X_{0}\right)$. Then for $i=0, \ldots, T$, the norm of $A_{r}$ on $V_{i}$ is bounded by $(r+1) e^{-r / p_{i}}$.

We have the decomposition

$$
L^{2}(X)=\operatorname{span}\{\pi\} \oplus \rho^{*} L_{0}^{2}\left(X_{0}\right) \oplus V_{0} \oplus V_{1} \oplus \ldots \oplus V_{T} .
$$

Decompose $b_{x_{0}, r_{0}}=\pi+u+v_{0}+\ldots+v_{T}$. For $i=1, \ldots, T$, denote $m\left(X, p_{i}\right)=\operatorname{dim} s V_{i}$. We have

$$
\begin{aligned}
\|u\|_{2}^{2} & =N^{-1}\left\|b_{x_{0}, r_{0}}\right\|_{2} \ll_{r_{0}} N^{-1} \\
\left\|v_{0}\right\|_{2}^{2} & \leq\left\|b_{x_{0}, r_{0}}\right\|_{2}^{2} \ll_{r_{0}} 1 \\
\left\|v_{i}\right\|_{2}^{2} & \leq N^{-1} m\left(X, p_{i}\right)\left\|b_{x_{0}, r_{0}}\right\|_{2} \ll_{r_{0}} N^{-1} m\left(X . p_{i}\right) .
\end{aligned}
$$


The first equality follow from Lemma 8.5, the second inequality is straightforward, and the third inequality follows from Lemma 8.6.

Then for $r=g\left(R_{X_{q}}\right)$,

$$
\left\|A_{r}\left(b_{x_{0}, r_{0}}-\pi\right)\right\|_{2}^{2}=\left\|A_{r} u\right\|_{2}^{2}+\left\|A_{r} v_{0}\right\|_{2}^{2}+\sum_{i=1}^{T}\left\|A_{r} v_{0}\right\|_{2}^{2} .
$$

Therefore one should prove that the RHS of Equation 8.4 is $O\left(N^{-1}\right)$.

Since $\|u\|_{2}^{2} \ll_{r_{0}} N^{-1}$ and $X_{0}$ has some $p_{0}\left(X_{0}\right)$ and the first summand of Equation 8.4 is $o\left(N^{-1}\right)$.

Since $\left\|v_{0}\right\|_{2}^{2} \ll_{r_{0}} 1$ and for some $\delta>2$, and $R$ large enough $g(R) \geq R+\delta \ln R$, the second summand Equation 8.4 is $o\left(N^{-1}\right)$.

For the third summand, we have

$$
\sum_{i=1}^{T}\left\|A_{r} v_{0}\right\|_{2}^{2} \leq N^{-1}(r+1)^{2} \sum_{i=1}^{T} e^{-2 r / p_{i}} m\left(X, p_{i}\right) m .
$$

This proves that if Inequality 8.1 holds then the third summand of Equation 8.4 is $o\left(N^{-1}\right)$.

Notice that for $1 \leq i \leq T, m\left(X, p_{i}\right)=M\left(X, p_{i}\right)-M\left(X, p_{i+1}\right)$, with $M\left(X, p_{T+1}\right)=0$. Then

$$
\begin{aligned}
\sum_{i=1}^{T}\left\|A_{r} v_{0}\right\|_{2}^{2} & \leq N^{-1}(r+1)^{2} \sum_{i=1}^{T} e^{-2 r / p_{i}} m\left(X, p_{i}\right) \\
& =N^{-1}(r+1)^{2} \sum_{i=1}^{T} e^{-2 r / p_{i}}\left(M\left(X, p_{i}\right)-M\left(X, p_{i+1}\right)\right) \\
& =N^{-1}(r+1)^{2}\left(M\left(X, p_{1}\right) e^{-2 r / p_{1}}+\sum_{i=2}^{T} M\left(X, p_{i}\right)\left(e^{-2 r / p_{i}}-e^{-2 r / p_{i-1}}\right)\right) \\
& \leq N^{-1}(r+1)^{2}\left(M\left(X, p_{1}\right) e^{-2 r / p_{1}}+\sum_{i=1}^{T} M\left(X, p_{i}\right) 2 r\left(p_{i}-p_{i-1}\right) e^{-2 r / p_{i}} p_{i-1}^{-2}\right),
\end{aligned}
$$

Where we used $\left(e^{-2 r / p_{i}}-e^{-2 r / p_{i-1}}\right)=2 r\left(p_{i}-p_{i-1}\right) e^{-2 r / p^{\prime}} p^{\prime-2}$, for some $p_{i-1} \leq p^{\prime} \leq p_{i}$.

By adding arbitrary $p_{i}$-s with $m\left(X, p_{i}\right)=0$ we may conclude

$$
\sum_{i=1}^{T}\left\|A_{r} v_{0}\right\|_{2}^{2} \leq N^{-1}(r+1)^{2}\left(\lim _{p_{i} \rightarrow 2, p_{i}>2} M\left(X, p_{i}\right) e^{-r}+2 r \int_{2}^{\infty} M(X, p) e^{-2 r / p} p^{-2} d p\right) .
$$

This proves that if Inequalities 8.2 and 8.3 hold then the third summand in Equation 8.4 is $o\left(N^{-1}\right)$.

9. Appendix I: Isoperimetric Inequalities and Concentration of Distance from a Fixed

\section{VERTEX}

The bounds we have allows us to prove the following isoperimetric inequality. Similar bounds are well known (see [9, Theorem 4.1]).

Lemma 9.1. Let $X=\Gamma \backslash \mathbb{H}$ be a quotient, and $p=p_{0}(X)$ as defined in Proposition 7.2. For $r \geq 0$, denote $\kappa_{r, p}=(r+1)^{2} e^{-2 r / p}$. For a closed set $Y \subset X$, let

$$
Y_{r}=\{x \in X \mid d(x, Y) \leq r\},
$$


and denote $c=\mu(Y) / \mu(X)$ and $c^{\prime}=\mu\left(Y_{r}\right) / \mu(X)$. Then

$$
c^{\prime} \geq \frac{c}{\left(\kappa_{r, p}(1-c)+c\right)}, \text { and hence also } c \leq \frac{\kappa_{r, p} c^{\prime}}{1-c^{\prime}+\kappa_{r, p} c^{\prime}} .
$$

Remark 9.2. For $c k_{r, p}^{-1}$ small $c^{\prime} \gg \frac{e^{2 r / p}}{(r+1)^{2}} c$. So for $p=2$, up to an $(r+1)^{-2}$ factor, the growth of small sets is the best possible, i.e. the size of the radius $r$-ball.

Remark 9.3. The result of [9, Theorem 4.1], which is more general and works for all surfaces, not necessarily hyperbolic, essentially replaces the exponent $2 / p=1-\sqrt{1-4 \lambda}$ by $\sqrt{\lambda}$, so the results above are asymptotically better for the relevant domain $0 \leq \lambda \leq 1 / 4$.

Proof. We may assume $\mu(Y)>0$. Let $b_{Y} \in L^{1}(Y)$ be defined by

$$
b_{Y}= \begin{cases}\mu^{-1}(x) & x \in Y \\ 0 & x \notin Y\end{cases}
$$

Then $\left\|b_{Y}\right\|_{1}=1,\left\|b_{Y}\right\|_{2}^{2}=\mu^{-1}(Y),\left\|A_{r_{0}} b_{Y}\right\|_{1}=1$ and $\operatorname{supp}\left(A_{r} b_{Y}\right) \subset Y_{r}$, so $\left\|A_{r} b_{Y}\right\|_{2}^{-2} \geq \frac{1}{\mu\left(Y_{r}\right)}$, i.e.

$$
\mu\left(Y_{r}\right) \geq\left\|A_{r} b_{Y}\right\|_{2}^{2}
$$

Decompose $b_{Y}=\pi+b$, with

$$
\|b\|_{2}^{2}=\left\|b_{Y}\right\|_{2}^{2}-\|\pi\|_{2}^{2}=\frac{1}{\mu(Y)}-\frac{1}{\mu(X)}=\frac{1-c}{\mu(Y)} .
$$

We have

$$
\begin{aligned}
\left\|A_{r_{0}} b_{Y}\right\|_{2}^{2} & =\left\|A_{r} b\right\|_{2}^{2}+\left\|A_{r} \pi\right\|_{2}^{2} \\
& \leq(r+1)^{2} e^{-2 r / p}\|b\|_{2}^{2}+\|\pi\|_{2}^{2} \\
& \leq(r+1)^{2} e^{-2 r / p}(1-c) \mu^{-1}(Y)+\mu^{-1}(X) \\
& =\left(\kappa_{r, p}(1-c)+c\right) \mu^{-1}(Y)
\end{aligned}
$$

Combining the two inequalities we get

$$
\mu\left(Y_{r}\right) \geq\left\|A_{r_{0}} b_{Y}\right\|_{2}^{-2} \geq \frac{c}{\left(\kappa_{r, p}(1-c)+c\right)} \mu(X) .
$$

The other inequality in the theorem follows from the first one.

We may now state the following concentration of distance theorem:

Theorem 9.4. There exists $a=a\left(p_{0}(X)\right)>0$ such that for each $x_{0} \in X$ there exists $R_{X, x_{0}}$ such that for every $\gamma>0$ :

$$
\mu\left(x \in X|| d_{X}\left(x, x_{0}\right)-R_{X, x_{0}} \mid \geq \gamma\right) / \mu(X) \ll_{p_{0}(X)} a^{-\gamma} .
$$

By Theorem 1.1 if $X$ is Ramanujan and $x_{0}$ has injectivity radius $r_{0}$ then $R_{X, x_{0}}$ satisfies $R_{X} \leq R_{X, x_{0}} \leq$ $\left.R_{X}+(2+\epsilon) \ln R_{X}\right)$.

Proof. For $r \geq 0$ denote

$$
Y(r)=\left\{x \in X \mid d\left(x, x_{0}\right) \leq r\right\}
$$


Choose $R_{X, x_{0}}$ to be such that

$$
\mu\left(Y\left(R_{X, x_{0}}\right)\right)=\frac{1}{2} \mu(X) .
$$

Let $Y=Y\left(R_{X, x_{0}}-\gamma\right)$. Then $Y_{\gamma}=Y\left(R_{X, x_{0}}\right)$ and

$$
\mu(Y) \mu(X)^{-1} \leq \frac{k_{\gamma, p} \frac{1}{2}}{1-\frac{1}{2}+k_{\gamma, p} \frac{1}{2}}=\frac{k_{\gamma, p}}{1+k_{\gamma, p}} \leq k_{\gamma, p} .
$$

Let $Z=Y\left(R_{X, x_{0}}+\gamma\right)$. Then $Y\left(R_{X, x_{0}}\right)_{\gamma}=Z$ and

$$
\mu(Z) \mu(X)^{-1} \geq \frac{\frac{1}{2}}{\left(\kappa_{r, p}\left(1-\frac{1}{2}\right)+\frac{1}{2}\right)}=\frac{1}{1+k_{\gamma, p}} .
$$

Hence

And finally,

$$
1-\mu(Z) \mu(X)^{-1} \leq \frac{k_{\gamma, p}}{1+k_{\gamma, p}} \leq k_{\gamma, p}
$$

$$
\begin{aligned}
\mu\left(x \in X:\left|d_{X}\left(x, x_{0}\right)-R_{X, x_{0}}\right| \leq \gamma\right) / \mu(X) & =1-\mu(Z) \mu(X)^{-1}-\mu(Y) \mu(X)^{-1} \\
& \leq 2 k_{\gamma, p} .
\end{aligned}
$$

We finish by noting that there exists $a=a(p)$ such that

$$
k_{\gamma, p} \ll_{p} a^{-\gamma} .
$$

\section{Appendix II: Comparison with the Flat Case}

In $[6$, Section $3 \mathrm{C}]$, Diaconis analyses the random walk on the Cayley graph of $\mathbb{Z} / N \mathbb{Z}$ with respect to the generators \pm 1 , and shows that it does not have a cutoff. Namely, he shows that the time $t_{0}^{T}$ until the random walk satisfies $\left\|p_{N}^{T}-\pi\right\|_{1} \leq e^{-T}$ is $\Theta\left(N^{2} T\right)$.

We will similarly analyze the Brownian random walk on the torus $a \mathbb{Z} \backslash \mathbb{R}$ where $a>0$, and show it does not have a cutoff as $a \rightarrow \infty$. Namely, we will show that time until the time $t_{0}^{T}$ until the random walk satisfies $\left\|p_{a}^{T}-\pi\right\|_{1} \leq e^{-T}$ is $\Theta\left(a^{2} T\right)$. Similar analysis shows that the Brownian random walk on quotients of $\mathbb{R}^{n}$ by $a \mathbb{Z}^{n}$ does not express a cutoff as $a \rightarrow \infty$.

It is also worth mentioning that the "distance $r_{1}$ " discrete random walk on $a \mathbb{Z} \backslash \mathbb{R}$ does not even converge in $L^{1}$ to the uniform probability, since it remains discrete. For higher dimensions the "distance $r_{1}$ " random walk does converge to the uniform probability (for reasons similar to Section 5), but does not express a cutoff by the central limit theorem and comparison with the Brownian motion.

Let $X_{a}=a \mathbb{Z} \backslash \mathbb{R}$ and let $x_{0} \in X$. The distribution of the Brownian random walk starting at $x_{0}$ at time $t$ for $x \in X$ is $p_{t}\left(x, x_{0}\right)=\left(\delta_{x_{0}} * f_{t}\right)(x)=\sum_{n \in \mathbb{Z}} f_{t}\left(x-x_{0}\right)$, with $f_{t}(x)=\frac{1}{\sqrt{2 \pi t}} \exp \left(-x^{2} / 2 t\right)$.

By normalizing and choosing $\lambda=a^{2}$, we may consider a fixed space $X=\mathbb{Z} \backslash \mathbb{R}$, a fixed point $x_{0}=\mathbb{Z} 0$ and let $f_{t}^{\lambda}(x)=\frac{1}{\sqrt{2 \pi \lambda^{-1} t}} \exp \left(-x^{2} \lambda / 2 t\right)$. Then $p_{t}^{\lambda}(x)=\sum_{n \in \mathbb{Z}} f_{t}^{\epsilon}(x+n)$.

Proposition 10.1. We have for every $\lambda>0, t \geq 0$.

$$
\exp \left(-\lambda^{-1} t\right) \leq\left\|p_{t}^{\lambda}-\pi\right\|_{1} \leq \sqrt{\frac{2}{1-\exp \left(-2 \lambda^{-1} t\right)}} \cdot \exp \left(-\lambda^{-1} t\right) .
$$


The proposition says that the time until $\left\|p_{t}^{\lambda}-\pi\right\|_{1} \leq e^{-T}$ takes place is $\Theta\left(T \cdot \lambda^{-1}\right)$. Therefore this random walk does not exhibit a cutoff.

Proof. Let us calculate the Fourier series of $p_{t}^{\lambda}$ :

$$
\begin{aligned}
\hat{p}_{t}^{\lambda}(m) & =\int_{0}^{1} p_{t}^{\lambda}(x) \exp (2 \pi i m x) d x= \\
& =\int_{0}^{1} \sum_{n \in \mathbb{Z}} f_{t}^{\lambda}(x+n) \exp (2 \pi i m x) d x \\
& =\int_{-\infty}^{\infty} f_{t}^{\lambda} \exp (2 \pi i m x) d x \\
& =\hat{f}_{t}^{\lambda}(m),
\end{aligned}
$$

where $\hat{f}_{t}^{\lambda}$ is the Fourier transform of $f_{t}^{\lambda}$. By a standard computation $\hat{f}_{t}^{\lambda}(\omega)=\exp \left(-\lambda^{-1} t \omega^{2}\right)$, so $\hat{p}_{t}^{\lambda}(m)=$ $\exp \left(-\lambda^{-1} t m^{2}\right)$.

On the one hand,

$$
\begin{aligned}
\left\|p_{t}^{\lambda}-\pi\right\|_{1} & \geq \int_{0}^{1}\left(p_{t}^{\lambda}(x)-1\right) \exp (2 \pi x) d x=\int_{0}^{1} p_{t}^{\lambda}(x) \exp (2 \pi x) d x \\
& =\hat{p}_{t}^{\lambda}(1)=\exp \left(-\lambda^{-1} t\right) .
\end{aligned}
$$

On the other hand,

$$
\begin{aligned}
\left\|p_{t}^{\lambda}-\pi\right\|_{2}^{2} & =\sum_{m \in \mathbb{Z}}\left(\hat{p}_{t}^{\lambda}(m)-\hat{\pi}(m)\right)^{2}=\sum_{m \in \mathbb{N} \backslash\{0\}}\left(\hat{p}_{t}^{\epsilon}\right)^{2}(m) \\
& =\sum_{m \in \mathbb{Z} \backslash\{0\}} \exp \left(-2 \lambda^{-1} t m^{2}\right) \\
& =2 \sum_{m=1}^{\infty} \exp \left(-2 \lambda^{-1} t m\right) \\
& \leq \frac{2}{1-\exp \left(-2 \lambda^{-1} t\right)} \exp \left(-2 \lambda^{-1} t\right) .
\end{aligned}
$$

Cauchy-Schwartz inequality completes the proof by

$$
\left\|p_{t}^{\epsilon}-\pi\right\|_{1} \leq\left\|p_{t}^{\epsilon}-\pi\right\|_{2} \leq \sqrt{\frac{2}{1-\exp \left(-2 \lambda^{-1} t\right)}} \exp \left(-\lambda^{-1} t\right) .
$$

\section{REFERENCES}

1. Robert Brooks and Eran Makover, Random construction of Riemann surfaces, Journal of Differential Geometry 68 (2004), no. 1, 121-157. 1

2. Valentina Cammarota, Alessandro De Gregorio, and Claudio Macci, On the asymptotic behavior of the hyperbolic Brownian motion, Journal of Statistical Physics 154 (2014), no. 6, 1550-1568. 4 
3. Giuliana Davidoff, Peter Sarnak, and Alain Valette, Elementary number theory, group theory and Ramanujan graphs, vol. 55, Cambridge University Press, 2003. 1

4. Edward B Davies and Nikolaos Mandouvalos, Heat kernel bounds on hyperbolic space and Kleinian groups, Proceedings of the London Mathematical Society 3 (1988), no. 1, 182-208. 4

5. Evan DeCorte and Konstantin Golubev, Lower bounds for the measurable chromatic number of the hyperbolic plane, arXiv preprint arXiv:1708.01081 (2017). 2

6. Persi Diaconis, Group representations in probability and statistics, Lecture Notes-Monograph Series 11 (1988), i-192. 10

7. ㄴ The cutoff phenomenon in finite Markov chains, Proceedings of the National Academy of Sciences 93 (1996), no. 4, 1659-1664. (document), 1

8. Joel Friedman, A proof of Alon's second eigenvalue conjecture, Proceedings of the thirty-fifth annual ACM symposium on Theory of computing, ACM, 2003, pp. 720-724. 1.5

9. Mikhail Gromov and Vitali D Milman, A topological application of the isoperimetric inequality, American Journal of Mathematics 105 (1983), no. 4, 843-854. 9, 9.3

10. Harish-Chandra, Spherical functions on a semisimple Lie group, I, American Journal of Mathematics (1958), 241-310. 1

11. Sigurdur Helgason, Groups and geometric analysis, volume 83 of Mathematical Surveys and Monographs, American Mathematical Society, Providence, RI (2000). 2

12. Peter Humphries, Density Theorems for Exceptional Eigenvalues for Congruence Subgroups, arXiv preprint arXiv:1609.06740 (2016). 2

13. Jonathan Huntley and Yonatan Katznelson, Density theorems for congruence groups in real rank 1, Duke Math. J 71 (1993), 463-473. 1, 1

14. MN Huxley, Exceptional eigenvalues and congruence subgroups, The Selberg Trace Formula and Related Topics, Contemp. Math 53 (1986), 341-349. 1

15. Henryk Iwaniec, Density theorems for exceptional eigenvalues of the Laplacian for congruence groups, Banach Center Publications 17 (1985), no. 1, 317-331. 1, 1, 2

16. Amitay Kamber, Lp-expander complexes, arXiv preprint arXiv:1701.00154 (2016). 1

17. — Lp-expander graphs, arXiv preprint arXiv:1609.04433 (2016). 7

18. Peter D Lax and Ralph S Phillips, The asymptotic distribution of lattice points in Euclidean and non-Euclidean spaces, Journal of Functional Analysis 46 (1982), no. 3, 280-350. 7

19. Eyal Lubetzky, Alex Lubotzky, and Ori Parzanchevski, Random walks on Ramanujan complexes and digraphs, arXiv preprint arXiv:1702.05452 (2017). 1

20. Eyal Lubetzky and Yuval Peres, Cutoff on all Ramanujan graphs, arXiv preprint arXiv:1507.04725 (2015). (document), 1

21. Alexander Lubotzky, Ralph Phillips, and Peter Sarnak, Ramanujan graphs, Combinatorica 8 (1988), no. 3, 261-277. 1

22. Alexander Lubotzky, Beth Samuels, and Uzi Vishne, Ramanujan complexes of type Ad, Israel Journal of Mathematics 149 (2005), no. 1, 267-299. 1

23. Adam Marcus, Daniel A Spielman, and Nikhil Srivastava, Interlacing families I: bipartite Ramanujan graphs of all degrees, Foundations of Computer Science (FOCS), 2013 IEEE 54th Annual Symposium on, IEEE, 2013, pp. 529-537. 1.5

24. Ori Parzanchevski and Peter Sarnak, Super-Golden-Gates for PU (2), arXiv preprint arXiv:1704.02106 (2017). 1

25. Peter Sarnak, Diophantine problems and linear groups, Proceedings of the International Congress of Mathematicians, vol. 1, 1990, pp. 459-471. 1, 8, 1

26. S_ Selberg's eigenvalue conjecture, Notices of the AMS 42 (1995), no. 11, 1272-1277. 1, 7

27. Peter Sarnak and Xiao Xi Xue, Bounds for multiplicities of automorphic representations, Duke Math. J 64 (1991), no. 1, 207-227. (document), 1, 1

28. Atle Selberg, On the estimation of Fourier coefficients of modular forms, Proc. Sympos. Pure Math, vol. 8, 1965, pp. 1-15. (document), 1, 1

29. Audrey Terras, Harmonic analysis on symmetric spaces - Euclidean space, the sphere, and the Poincaré upper half-plane, Springer Science \& Business Media, 2013. 2, 2.1, 2 